\title{
Exosomal miRNAs in tumor microenvironment
}

\author{
Shiming Tan ${ }^{1 \dagger}$, Longzheng Xia ${ }^{1 \dagger}$, Pin Yi ${ }^{1,2}$, Yaqian Han ${ }^{1}$, Lu Tang ${ }^{1,2}$, Qing Pan ${ }^{1,2}$, Yutong Tian ${ }^{1,2}$, Shan Rao , \\ Linda Oyang ${ }^{1}$, Jiaxin Liang ${ }^{1}$, Jinguan Lin', Min Su', Yingrui Shi', Deliang Cao ${ }^{1,3}$, Yujuan Zhou ${ }^{1 *}$ and Qianjin Liao ${ }^{1 *}$
}

\begin{abstract}
Tumor microenvironment (TME) is the internal environment in which tumor cells survive, consisting of tumor cells, fibroblasts, endothelial cells, and immune cells, as well as non-cellular components, such as exosomes and cytokines. Exosomes are tiny extracellular vesicles (40-160nm) containing active substances, such as proteins, lipids and nucleic acids. Exosomes carry biologically active miRNAs to shuttle between tumor cells and TME, thereby affecting tumor development. Tumor-derived exosomal miRNAs induce matrix reprogramming in TME, creating a microenvironment that is conducive to tumor growth, metastasis, immune escape and chemotherapy resistance. In this review, we updated the role of exosomal miRNAs in the process of TME reshaping.
\end{abstract}

Keywords: exosomal miRNAs, tumor microenvironment (TME), CAFs, angiogenesis, immune microenvironment

\section{Background}

TME is a complex ecosystem and an important player in all stages of tumorigenesis. TME consists of cancer cells, cancer-associated fibroblasts (CAFs), endothelial cells, immune cells, extracellular matrix (ECM), microvessels, and biomolecules infiltrated [1-5]. Compared with the normal internal environment, TME is more prominently characterized by hypoxia. Hypoxia caused by rapid appreciation of tumor cells leads to release of matrix metalloproteinases (MMPs), hypoxia inducible factor-1 $\alpha$ (HIF-1 $\alpha$ ), vascular endothelial growth factor (VEGF) and other stimulating factors. Reshaping TME provides a niche for interaction between tumor cells and surrounding fibroblasts, endothelial cells, and immune cells [4, 6-9]. These cells interact with tumor cells through TME to induce a variety of biological events, such as appreciation, migration, angiogenesis,

\footnotetext{
*Correspondence: march-on@126.com; yujany_zhou@163.com

†Shiming Tan and Longzheng Xia contributed equally to this work.

${ }^{1}$ Hunan Key Laboratory of Translational Radiation Oncology, Hunan Cancer Hospital and The Affiliated Cancer Hospital of Xiangya School of Medicine, Central South University, 283 Tongzipo Road, Changsha 410013, Hunan, China

Full list of author information is available at the end of the article
}

immunosuppression, and drug resistance for tumor development [10-14].

MicroRNAs (miRNAs) are a class of short ncRNAs with 19-25 nucleotides in length [15]. Through regulating gene expression, miRNAs regulate a variety of important biological functions, such as proliferation, apoptosis, differentiation, migration, invasion and drug resistance. Genetic or epigenetic changes in cancer cells can induce abnormal expression of miRNAs, thus causing abnormal expression of their target genes [16-21]. miRNAs function through 6-7 base complementary binding to target mRNA and inhibition of target gene expression at the level of protein [22-24]. From the literature, miRNAs can work as oncogenes to promote the formation and biological changes of TME [25-28]. For example, miR-9 and miR-200s induce normal fibroblasts (NFs) in TME to transform into CAFs and promote tumor metastasis $[29,30]$, miR-526b and miR-655 promote angiogenesis and lymphangiogenesis in TME [31], and miR-340-5p and miR-561 induce formation of immunosuppressive microenvironment [32, 33]. How these biologically active miRNAs are transmitted and function

(c) The Author(s). 2020 Open Access This article is licensed under a Creative Commons Attribution 4.0 International License, which permits use, sharing, adaptation, distribution and reproduction in any medium or format, as long as you give appropriate credit to the original author(s) and the source, provide a link to the Creative Commons licence, and indicate if changes were made. The images or other third party material in this article are included in the article's Creative Commons licence, unless indicated otherwise in a credit line to the material. If material is not included in the article's Creative Commons licence and your intended use is not permitted by statutory regulation or exceeds the permitted use, you will need to obtain permission directly from the copyright holder. To view a copy of this licence, visit http://creativecommons.org/licenses/by/4.0/ The Creative Commons Public Domain Dedication waiver (http://creativecommons.org/publicdomain/zero/1.0/) applies to the data made available in this article, unless otherwise stated in a credit line to the data. 
in cells and TME is an important breakthrough in the study of TME.

Recently, exosomes are considered to be the key mediators responsible for the heterogeneity of the TME and carry biologically active cargos, such as protein, metabolites, nucleic acids (e.g. ncRNAs), to shuttle between tumor cells and TME, thereby affecting tumor development [34-37]. Among the biologically active substances, tumor-derived exosomal miRNAs can induce TME heterogeneity while changes in TME promote tumor progression. This paradigm, similar to a positive feedback loop, makes the uncontrollable growth of the tumor [38-43]. In this article we updated the interaction of exosomal miRNAs and TME.

\section{The overview of microenvironment and exosomes in cancer}

\section{The components of tumor microenvironment}

Growth, metastasis and treatment resistance of tumors are inseparable from the support of TME, a dynamic ecosystem containing multiple cell types and noncellular components. Some of the basic biological behavioral features of tumors, such as proliferation, migration, invasion, apoptosis inhibition, immune evasion, angiogenesis, and metabolic reprogramming are all affected by TME. The complex communication network in TME is the basis for the regulation of these biological functions, including autocrine and paracrine. Exocrine-mediated communication is an important emerging pathway in paracrine signal transduction [2].

Non-tumor cells in TME, such as fibroblasts, endothelial cells and immune cells, are affected by tumor-related active substances, and their original cellular functions undergo tumor-like changes, constantly adapt to new environments and promote tumor growth. Due to the influence of TME, NFs are activated into CAFs. CAFs are the most abundant stromal cells in TME, producing an ECM that differs from normal ECM in terms of stiffness and alignment, which support tumor cells migration [9]. Hypoxia in TME causes tumor to secrete angiogenic factors to act on endothelial cells and promote angiogenesis $[44,45]$. The immune cells in TME show diversity, and they block the immune response. The inflammatory molecules around the tumor cells also cause the system to fail to recognize and eliminate cancer cells $[38,46,47]$. These make TME a complex heterogeneous environment and often leads to an uncontrollable trend in the development of tumors $[48,49]$.

\section{The biosynthesis and function of extracellular vesicles and exosomes}

Extracellular vesicles (EVs) are nano-sized lipid bilayer vesicles $(40-1000 \mathrm{~nm}$ in diameter) released by cells or detached from the plasma membrane [50, 51]. EVs are generally divided into two categories: ectosomes and exosomes. Ectosomes are vesicles formed from the plasma membrane sprouting outwards, including microvesicles, microparticles and large vesicles with a size range of $50-1000 \mathrm{~nm}$ in diameter. Exosomes are small extracellular vesicle (sEVs) in a size range of $40-160 \mathrm{~nm}$ in diameter with an endosomal origin. EVs have biological activities and mediate intercellular communication [36]. During tumor progression, EVs derived from different cells (tumor cells, stromal cells, immune cells, etc.) play an important role and participate in the formation of TME [44, 52-54].

In this review, we mainly focused on the exosomes. However, because of absence of strict standards for exosome isolation and purification methods, the International Society for Extracellular Vesicles encouraged researchers to establish minimum requirements and strictly control the integrity, size, molecular cargo, and functionality of the vesicle population [38, 55-57], so that we narrowed the research of exosomes based on the widely accepted methods. Exosomes are small extracellular vesicles (40-160 $\mathrm{nm}$ in diameter) formed by dynamic exocytosis [58-60]. Exosomes originate from the luminal cavity or early intracellular bodies in the circulation pathway of the plasma membrane. These membranes or early intracellular bodies will sag inward to form intraluminal vesicles (ILV), which will further develop into multivesicular bodies (MVB) [61, 62]. In general, multivesicular bodies are fused with lysosomes to be degraded, but some multivesicular bodies are fused to the cell surface under the traction of intracellular molecular motors and eventually secreted outside the cell, which called exosomes [36, 63].

Exosomes are involved in the biology of many diseases. Exosomes can regulate the immune response and inflammation, possibly through transfer and presentation of antigen peptides, to induce expression of inflammatory genes in recipient cells $[64,65]$. In metabolism and cardiovascular diseases, exosomes induce metabolic disorders in adipocytes and islet cells [66, 67]. Exosomes may impair the formation of neurotoxic oligomers and promote neurodegeneration [68-70]. More importantly, exosomes are associated with tumor growth, angiogenesis, metastasis, sensitivity to chemotherapy, and immune evasion $[47,71,72]$.

\section{miRNAs sorting to exosomes}

Exosomes contain a variety of biologically active molecules, such as proteins, lipids and nucleic acids. miRNAs are one of them and play an important role in intercellular cellular transport and signal transduction [73-75]. Exosomes can transfer metabolites and promote communication between different cells through the exchange of exosomal miRNAs, and then play an immune response, 
tumor microenvironment remodeling and tumor metastasis during tumor progression [38, 76-78]. Many reports indicate that exosomes affect the biology of recipient cells by transferring miRNAs from donor cells to recipient cells, but the mechanism of how exosomes sorting miRNAs has not been thoroughly solved. According to exosomes database (www.exocarta.org), 2838 miRNAs are listed in the latest update. Among the 2588 annotated miRNAs in the human genome, 593 miRNAs have been detected in exosomes [79]. Four potential mechanisms for sorting miRNAs into exosomes were proposed. The neural sphingomyelinase 2 (nSMase2) was the first molecule found to be linked with miRNAs packaging into exosomes. Overexpression of nSMase2 leads to an increased number of miRNAs loaded into exosomes. This suggests that the neural sphingomyelinase 2 (nSMase2)dependent pathway is associated with the sorting of exosomal miRNAs [80]. The second is based on the control of the sumoylated form of heterogeneous nuclear ribonucleoprotein (hnRNP). Sumoylated hnRNPA2B1 controls the sorting of exosomal miRNAs by recognizing the GGAG and GGCU base sequence of the 3 'end region of miRNAs $[81,82]$. The third is that most exosomal miRNAs isolated from urine or B cells were uridylated at 3 ' ends. The sorting of miRNAs to ILV may also require hydrophobic modification and GGAG base sequence at 3 ' end of the miRNAs. This indicates that the 3 'ends of miRNAs may be involved in directing miRNAs into exosomes [83, 84]. Finally, there are reports that Argonaute proteins (functional carriers of miRNAs) are related to the selection of exosomal miRNAs. Knocking out Argonaute 2 (Ago2) reduces the contents of certain exosomal miRNAs, such as miR-142-3p, miR-150, and miR-451 [85, 86]. In summary, some specific protein complexes and miRNAs own structural characteristics have affects the miRNAs' transfer to exosomes, but the complete sorting mechanism and process have not yet been elucidated and need further exploration.

\section{The role of exosomal miRNAs in TME}

During the progression of the tumor, primary tumorderived exosomal miRNAs can be transferred to nonmalignant cells in the tumor microenvironment to induce heterogeneity [50, 87-89]. At the same time, with the changes in biological activity of non-malignant cells in the tumor microenvironment, non-malignant cells can also secrete exosomal miRNAs to further regulate tumor cells or other microenvironmental components [40, 90]. In most studies, the stromal cell receptors of cancer-derived exosomal miRNAs are cancer-associated fibroblasts (CAFs), endothelial cells and immune cells dynamically regulate each other in TME. Exosomal miRNAs on the heterogeneity of TME is mainly reflected in the fact that exosomal miRNAs can activate cancer-associated fibroblasts and thus reshape ECM, which is beneficial to the spread of tumor cells. Exosomal miRNAs promote endothelial cells to form tubes, and the formation of abundant vascular networks is conducive to the metabolism and survival of tumor cells. Exosomal miRNAs also mediate inflammatory cell infiltration and immune escape, which is conducive to colonization and proliferation of tumor cells. Through these macroscopic effects, exosomal miRNAs can make TME more suitable for tumor development [91]. Herein, we focused on the roles of exosomal miRNAs in following aspects.

\section{Reshaping ECM to promote tumor progression}

Extracellular matrix (ECM) is composed of protein and carbohydrates, with the functions of connection, support, water retention, anti-stress and protection. ECM supports the basic life activities of cells, such as proliferation, differentiation, and migration [92, 93]. However, tumors are often accompanied by dysfunction of ECM. Tumor development is a complex process involving dynamic interactions between malignant cells and their surrounding stroma composed of cells and non-cellular components. Within the stromal, fibroblasts represent not only the major cell types, but also the main source of extracellular matrix (ECM) and soluble factors [94, 95]. Normal fibroblasts exert multiple inhibitory functions against cancerinitiating and metastasis through direct cell-cell contact, paracrine signaling, and ECM integrity [96]. However, tumor-derived exosomal miRNAs can trigger a series of tumor-promoting signals, inducing normal fibroblasts (NFs) transformation into CAFs, which changes the original ECM physiological state, thus creating the optimal niche for the widespread growth of cancer cells [96, 97].

In tumors, tumor cell-derived exosomal miRNAs are highly diverse and are capable of differentiating NFs into CAFs through a variety of signaling pathways. Exosomal miRNAs from cancer cells elicit a parenchymal signaling response at the receptor site and effectively inducing fibroblast activation, such as $\alpha$-smooth muscle actin $(\alpha$ SMA), fibroblast growth factor 2 (FGF2) and fibroblast activating protein (FAP) expression [98-100]. Matrix composed of CAFs is conducive to the proliferation and migration of tumor cells. In ovarian cancer, the cancerrelated exosomal miR-124 targets sphingosine kinase 1 (SPHK1) and upregulates $\alpha$-SMA and FAP, which differentiates NFs into CAFs and regulates CAFs migration and invasion [101, 102]. High expression of exosomal miR-27b-3p and miR-214-3p in myeloma cells triggers proliferation and apoptotic resistance of bone marrow fibroblasts via the FBXW7 and PTEN/AKT/GSK3 pathways. At the same time, miR-27-3p and miR-214-3p were up-regulated in fibroblasts co-cultured with myeloma, and activated expression of fibroblast activation markers $\alpha$-SMA and FAP. The biological behavior of 
bone marrow fibroblasts is programmed to alter the myeloma microenvironment [103, 104]. Exosomal miRNAs in digestive system tumors also reshaped ECM in adjacent sites and promote tumor progression. Exosomal miR-27a derived from gastric cancer (GC) is transported to fibroblasts, and thus results in decreased expression of CSRP2, enhanced expression of $\alpha$-SMA, and differentiation of fibroblasts into CAFs [105]. Exosomal miR-10b secreted by colorectal cancer cells can be transferred to fibroblasts, where it inhibits PIK3CA expression and PI3K/ Akt/mTOR pathway activity, promote expression of TGF$\beta$ and $\alpha$-SMA, and enable fibroblasts to acquire the characteristics of CAFs $[106,107]$. These changes promote the proliferation, migration and invasion of tumor cells. Exosomal miRNAs have found similar effects in colorectal cancer (CRC). Exosomal miR-2149-5p, miR-6737-5p, and miR-6819-5p can inhibit the expression of TP53 in fibroblasts to promote tumor proliferation [108].

In addition, changes in ECM also affect angiogenesis, inflammatory response, and metabolic reprogramming. Phenomenon was shown in melanoma where highly expressed exosomal miR-155 inhibits the expression of SOCS1, activates the JAK2/STAT3 pathway, up-regulates the expression of FGF2, VEGFA and MMP9 in CAFs, and promotes the formation of blood vessels in the tumor $[109,110]$. In hepatocarcinoma (HCC), exosomal miR-21 is transferred to CAFs, directly targeting PTEN to activate
PDK1/Akt signaling, up-regulating VEGF, MMP2, MMP9, bFGF, and TGF-beta and thus promoting angiogenesis [111, 112]. Exosomal miR-1247 targets B4GALT3 and activates the beta1-integrin-NF-kappaB signaling pathway, which activates CAFs to secrete the inflammatory cytokines IL- 6 and IL-8 and induce inflammatory infiltration [113]. Exosomal miR-9 and miR-105 are derived from breast cancer; the former promotes the activation of NFs into CAFs by affecting MMP1, EFEMP1 and COL1A1 [30], and the latter activates MYC signal transduction to induce metabolic reprogramming of CAFs, and adapts CAFs to different metabolic environments, promoting tumor growth [18]. Similar reports include miRNA-142$3 p$ in EVs secreted by lung cancer cells, which promotes the cancer phenotype of lung fibroblasts [114] (Fig. 1 and Table 1).

These researches show that cancer-derived exosomal miRNAs can affect the physiological function of stroma. Conversely, a reciprocal exosomal miRNAs exchange from the stroma to cancer cells also modulates cancer progression. For example, CAFs-derived exosomal miR$148 \mathrm{~b}$ in the matrix surrounding endometrial cancer can up-regulate DNMT1, leading to changes in EMT-related molecules like E-cadherin, $\mathrm{N}$-cadherin, vimentin, and fibronectin and promoting cancer cell metastasis [65]. CAFs are resistant to cisplatin and deliver exosomal miR-196a, which binds to target CDKN1B and ING5,

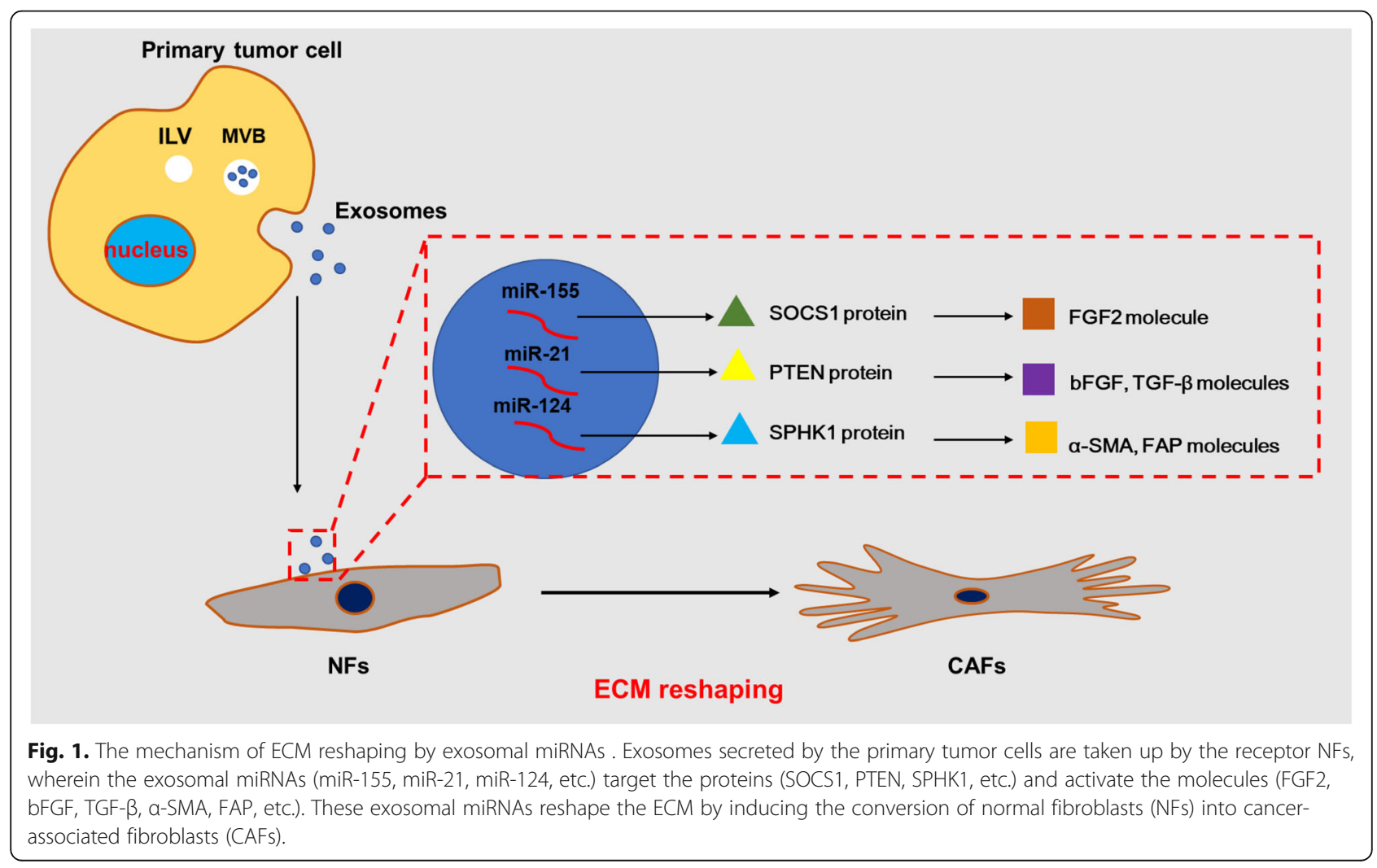




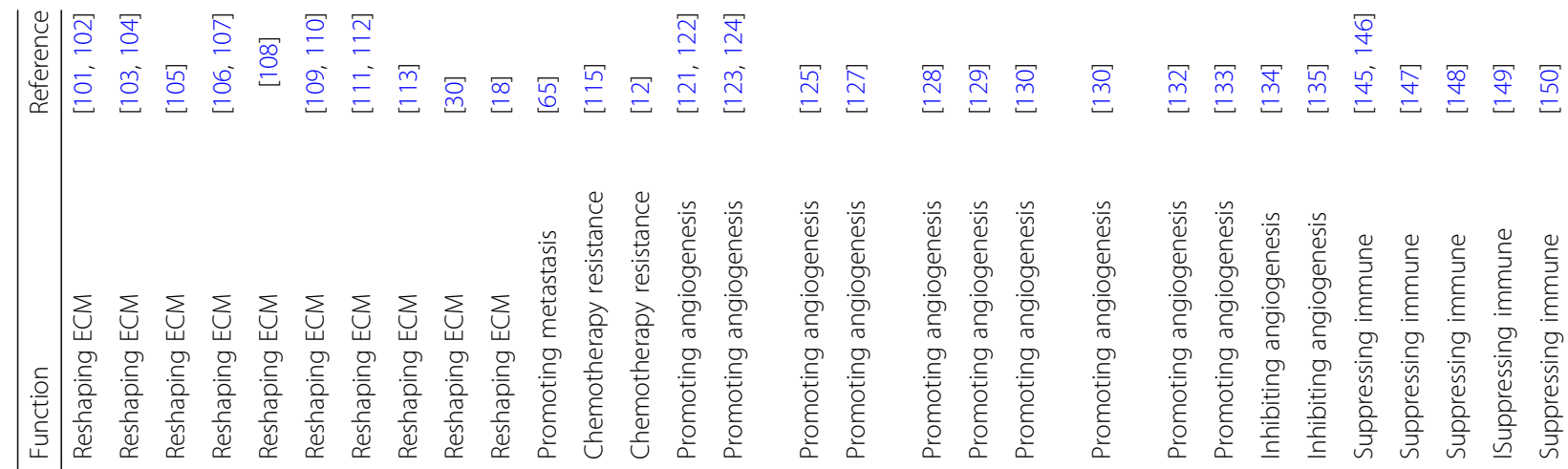

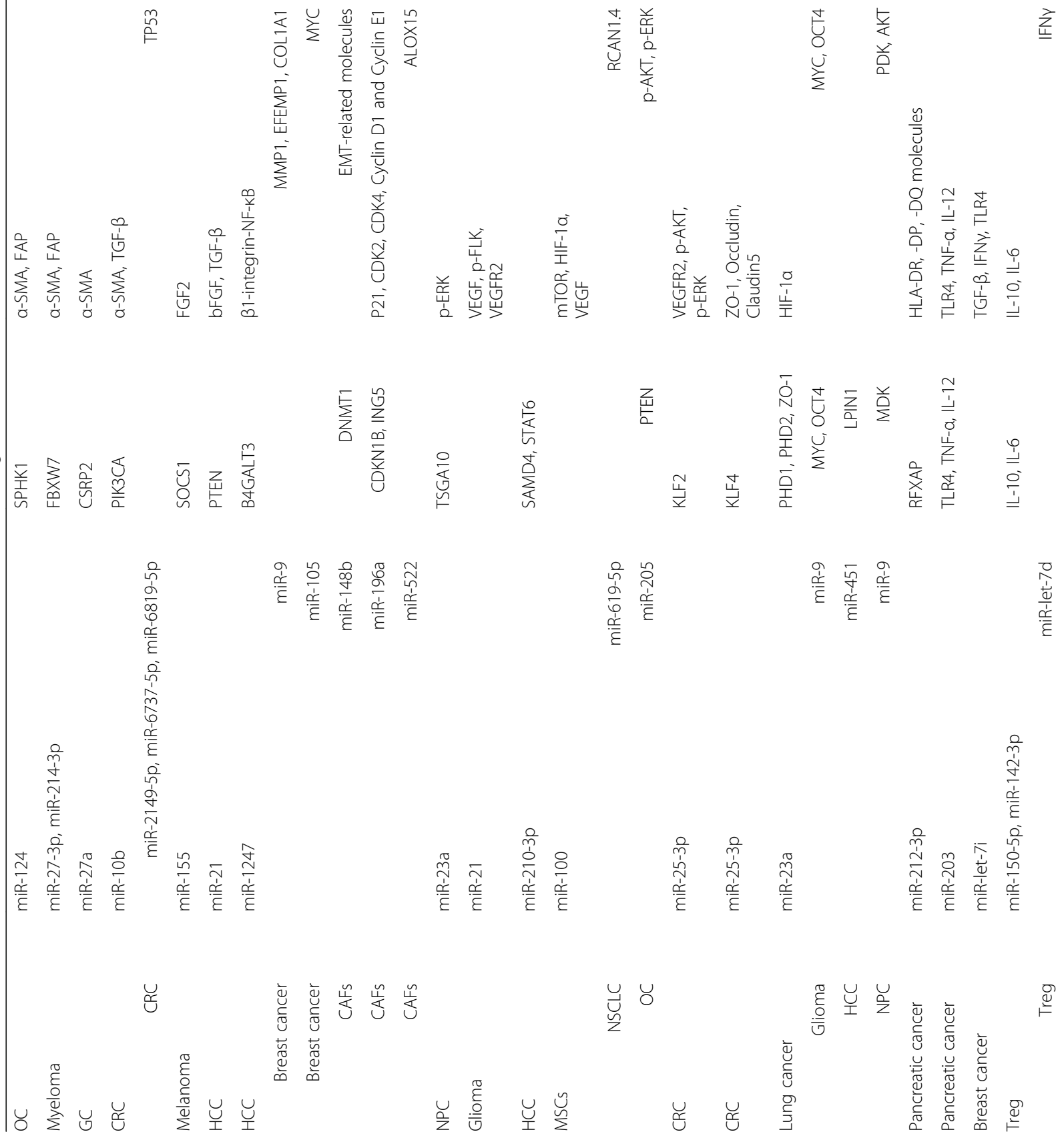

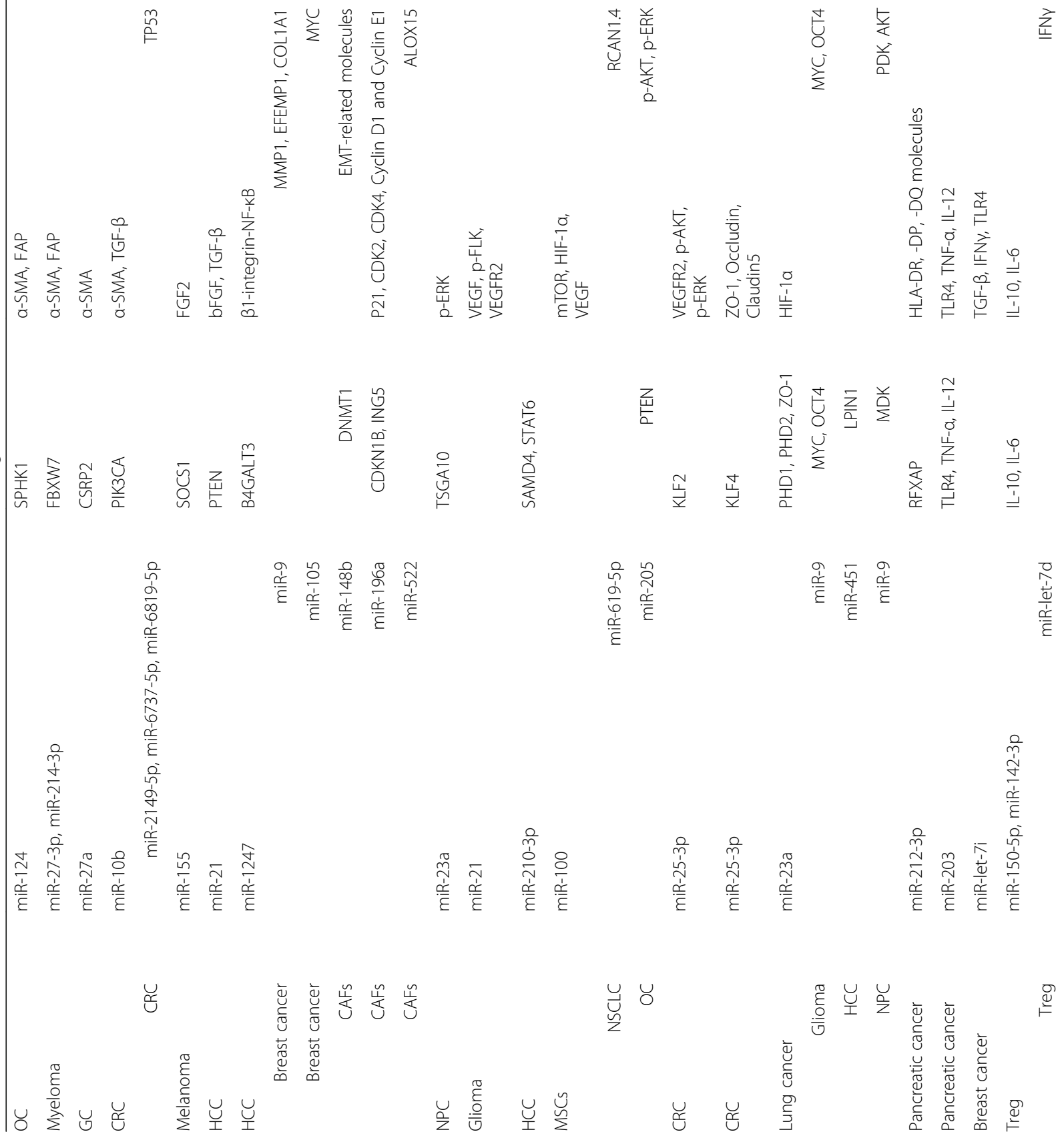




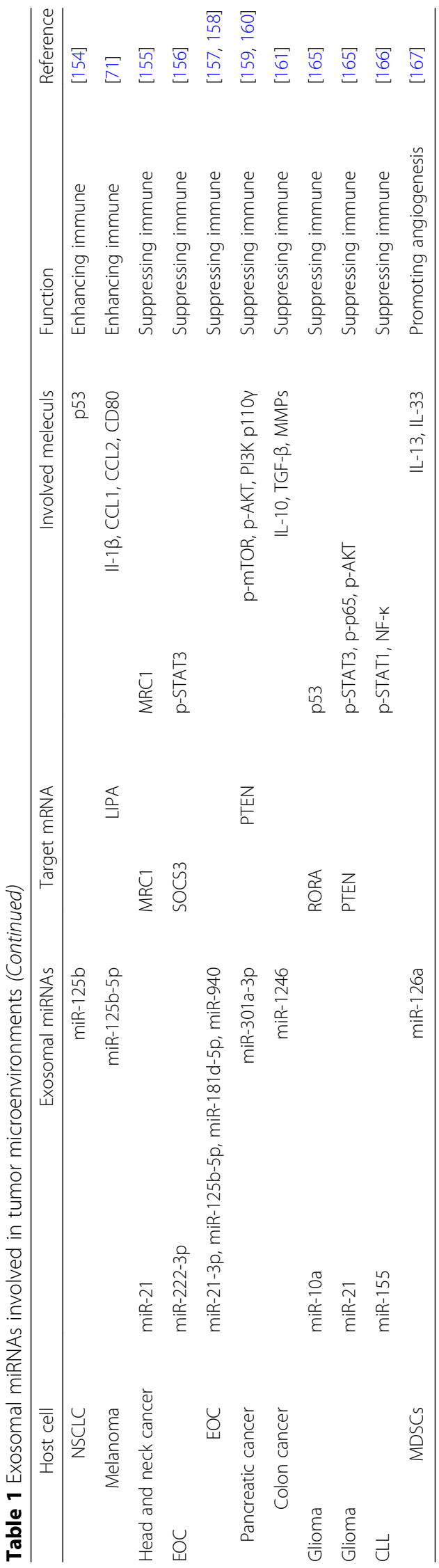


mediates the expressions of p27, CDK2, CDK4, Cyclin D1 and Cyclin E1 and thus induces cisplatin resistance to cancer cells [115]. CAFs-derived exosomal miR-522 reduces the contents of lipid-ROS in gastric cancer cells by inhibiting the expression of ALOX15, which leads to a decrease in the sensitivity of gastric cancer to chemotherapy [12].

Compared with NFs, CAFs have the characteristics of excessive proliferation and unique cytokines. This not only induces the formation of new blood vessels, but also promotes the entry of immune cells into TME, which greatly changes the physiological function of ECM to support tumor proliferation, metastasis and treatment resistance [116, 117]. However, cells involved in ECM formation are not only fibroblasts, but also chondrocytes, osteoblasts, and certain epithelial cells. Exosomal miRNA remodeling of ECM can also be achieved by affecting the function of these cells. For example, studies have shown that cancer-secreted exosomal miR-940 promotes osteogenic differentiation of mesenchymal cells by targeting ARHGAP1 and FAM134A, and then induces osteogenic phenotypes in the bone metastasis microenvironment and promotes tumor metastasis [118]. But research on the interaction of exosomal miRNAs with these cells is not comprehensive. At the same time, the composition of ECM not only includes collagen (synthesized by fibroblasts, chondrocytes, osteoblasts and certain epithelial cells and secreted outside the cell), but also includes non-collagen glycoproteins, glycans and elastin. Whether exosomal miRNAs reshape ECM by affecting these ingredients remains to be proven.

\section{Promoting angiogenesis to enhance proliferation and migration}

Tumor growth depends to a large extent on the metabolism of cancer cells [119]. The disordered distribution of tumor blood vessels and the loss of normal vascular function lead to local hypoxia and impaired nutrient supplies. At the same time, the distance gradient between different vascular beds also leads to the imbalance of drug distribution and absorption [120]. These changes of vascular network promote the formation of internal microenvironment and intratumoral heterogeneity.

The exosomal miRNAs can be taken up by the vascular endothelial cells to change the original distribution and physiological functions of the blood vessels in the microenvironment. Exosomal miRNAs secreted by tumor cells have been reported to promote angiogenesis in TME. In nasopharyngeal carcinoma (NPC), exosomal miR-23a mediates angiogenesis by repressing TSGA10 and phosphorylation of ERK, which enhances tube generation ability of human umbilical vein endothelial cells (HUVECs) in vitro and in vivo [121, 122]. Glioma stem cell-derived exosomal miR-21 stimulates VEGF/p-FLK/
VEGFR2 signaling pathway to promote angiogenesis in endothelial cells $[123,124]$. The exosomal miR-210-3p secreted by HCC cells is transferred to endothelial cells, targeting SMAD4 and STAT6 to promote angiogenesis, and it is found that the higher miR-210-3p in the serum of HCC patients is positively correlated with the microvessel density in HCC tissues [125]. EVs and sEVsmediated miRNAs transfer also promotes angiogenesis in TME. In NSCLC, EVs-mediated miR-142-3p transferred to endothelial cells and fibroblasts, inhibiting the expression of TGF $\beta$ R1, PDGFR- $\beta$ and $\mathrm{p}-\mathrm{SMAD} 2 / 3$ to promote angiogenesis [114]. Human ovarian carcinoma cell line SKOV-3 secretes miR-141-3p in small extracellular vesicles (sEVs), which activates the JAK-STAT3 pathway in endothelial cells and promotes angiogenesis [126]. Besides, exosomal miRNAs that promote angiogenesis can also be derived from other cells. Exosomal miR-100 from human mesenchymal stem cells (MSCs) affects the mTOR/HIF-1 $\alpha$ /VEGF signaling axis to promote angiogenesis in breast cancer [127].

The rich vascular network in TME is beneficial to the proliferation and metastasis of cancer cells. Exosomal miR-619-5p inhibits the expression of RCAN1.4, promotes angiogenesis, and facilitates the growth and metastasis of cancer cells [128]. Recent studies have shown that circulating exosomal miR-205 expression is elevated in OC patients and is related to microvessel density, and exosomal miR-205 induces angiogenesis via the PTENAKT pathway, and promotes tumor cell proliferation in vitro [129]. Changes in the vascular microenvironment are not only in the number of blood vessels, but also in vascular permeability, adhesion, and ability to form a ring. The colorectal cancer-derived exosomal miR-25-3p can down-regulate KLF2 and KLF4, and KLF2 affects the tube formation ability of HUVECs through the VEGFR2/p-Erk/p-Akt pathway while KLF4 activates ZO-1/Occludin/Claudin5 pathway to affect the growth of the aortic rings, which in turn changes the vascular microenvironment [130, 131]. Under hypoxic conditions, lung cancer cell-derived exosomal miR-23a directly inhibits prolyl hydroxylase 1 and 2 (PHD1 and PHD2) and accumulates HIF- $1 \alpha$ in endothelial cells, inducing angiogenesis, and exosomal miR- 23a also inhibits ZO-1, increasing vascular permeability and transendothelial migration of cancer cells [132]. In human glioma, exosomal miR-9 promotes angiogenesis, vascular permeability and adhesion through the MYC/ OCT4 pathway [133] (Fig. 2).

Exosomal miRNAs influence on vascular network is not only promotion, but sometimes also play an inhibitory effect. Studies have found that exosomal miR-451 acts as a tumor suppressor and targets LPIN1 to induce apoptosis both in HCC cell lines and HUVECs. In addition, miR-451a suppresses HUVECs tube formation 


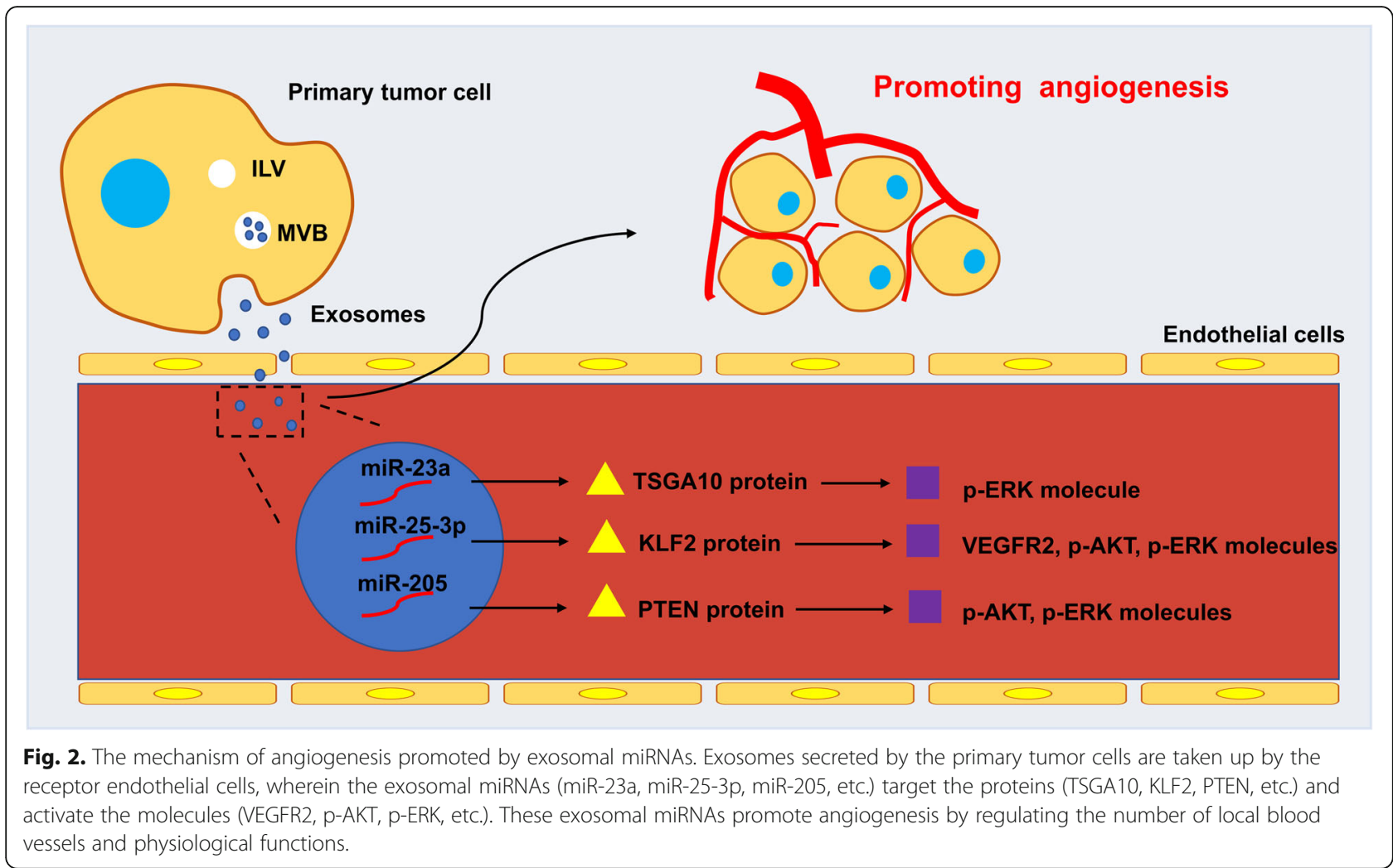

and vascular permeability [134]. NPC-derived exosomal miR-9 up-regulates MDK and activates the PDK/Akt signaling pathway to inhibit the formation of endothelial cells. High expression of MDK in NPC tumor samples is positively correlated with microvessel density, revealing the anti-angiogenic effects of exosomal miR-9 in the development of nasopharyngeal carcinoma [135]. Except for tumor-derived exosomal miRNAs, which inhibit angiogenesis, non-tumor cells have similar functions. miR-15a, miR-181b, miR-320c, and miR-874 in EVs released by human liver stem-like cells (HLSCs) possess an anti-tumorigenic effect by inhibiting tumor angiogenesis [136]. According to these reports, it can be found that exosomal miRNAs can regulate the vascular network in TME through multiple signaling pathways, but these molecular mechanisms have not been fully elucidated and need to be explored in the future.

\section{Promoting the formation of immunosuppressive environment}

In the TME, immune cells including lymphocytes, dendritic cells, and macrophages, regularly infiltrate tumor tissues and adjacent sites. Through multiple signal transduction pathways mediated by exosomal miRNAs, tumor cells can inhibit the maturation and differentiation of immune cells, thereby creating an immune microenvironment suitable for tumor growth [41, 137, 138]. At the same time, in hypoxia and low nutrient supplies in the microenvironment, tumor cells often secrete metabolic by-products, such as lactic acid, nitric oxide, reactive oxygen species, prostaglandins and arachidonic acid, leading to the formation of an inflammatory microenvironment [139, 140]. Changes in the biological functions of various immune cells in microenvironment and the production of inflammatory mediators result in tumor cell escaping from immune surveillance.

Dendritic cells (DCs) are the most powerful professional antigen presenting cells in the body. Mature DCs can effectively activate the initial $\mathrm{T}$ cells and maintain the central part of the immune response [141, 142]. Tumor-derived and endogenous exosomal miRNAs can regulate cross-presentation in dendritic cells and with other immune cells, this exomsomal miRNAs-mediated intercellular communication may affect the maturation of DCs [143, 144]. In pancreatic cancer, exosomal miR212-3p targets MHC class II TF RFXAP resulting in reduced expression of HLA-DR, -DP, and -DQ molecules and thus interfering with the function of DCs cells [145, 146]. Exosomal miR-203 is able to reduce the expression of TLR4, TNF- $\alpha$ and IL-12 in DCs, affecting the activation of natural killer cells (NKs) [147]. Up-regulated exosomal miR-let-7i in tumor-derived exosomes (TEX) can be taken up by mDCs, resulting in changes in intracellular levels of IL-6, IL-17, IL-1b, TGFbeta, SOCS1, KLRK1, IFN $\gamma$, and TLR4, thereby suppressing the immune response [148]. miRNAs from regulatory $\mathrm{T}$ cells (Treg) 
can also affect the immune response, EVs-mediated miR-150-5p and miR-142-3p can be transferred to DCs to induce a cell-refractory phenotype, resulting in increased IL-10 and decreased IL-6 expression [149], exosomal miR-let-7d is transferred to $\mathrm{T}$ helper 1 (Th1) cells to inhibit Th1 cells proliferation and IFNY secretion, and IFNY secreted by Th1 cells (a subtype of Naïve CD4 T cells) plays a central role in anti-tumor immunity [150].

Tumor-associated macrophages (TAMs) are one of the most abundant immune cells in TME. TAMs play a huge role in the proliferation and migration of tumor cells and counteract the cytotoxic effect of $\mathrm{T}$ lymphocytes and NKs, facilitating cancer cells to evade immune surveillance $[140,151]$. TAMs have strong plasticity and can differentiate into immune-stimulating (M1-polarized) TAMs or oppositely immune-suppressive (M2-polarized) TAMs, respectively, having different biological functions [152]. TAMs in tumors often behave as M2 phenotype and are usually associated with poor prognosis [153]. A large number of studies have reported that exosomal miRNAs can regulate the phenotypes of TAMs. Exosomal miR-125b derivied from lung adenocarcinoma cells promotes macrophage repolarization toward an antitumor M1 phenotype [154]. Exsomal miR-125b-5p secreted by melanoma cells targets LIPA and increases the expression of M1 phenotype markers IL-1 $\beta$, CCL1, CCL2, and CD80 [71]. Oppositely, exosomal miR-21 taken up by $\mathrm{CD} 14^{+}$human monocytes inhibits the expression of the M1 marker and increases the expression of the M2 marker. Knockout of miR-21 in Snail-expressing human head and neck cancer cells attenuated M2 polarization of TAMs, and miR-21 was found to be positively correlated with M2 marker MRC1 in head and neck cancer tissues [155]. In epithelial ovarian cancer (EOC), exosomal miR222-3p can be transferred to macrophages, downregulating SOCS3, inducing phosphorylation of STAT3, and thus leading to polarization of the M2 macrophages [156]. In hypoxia, EOC-derived exosomal miR-21-3p, miR-125b-5p, miR-181d-5p, and miR-940 differentiate TAMs into M2 phenotypes and promote tumor progression $[157,158]$. Likewise, exosomal miR-301a-3p derived from hypoxic pancreatic cancer cells activates the PTEN/ $\mathrm{PI} 3 \mathrm{~K} \gamma$ signaling pathway to trigger M2 phenotype polarization of macrophages $[159,160]$. Mutant p53 colon cancer cells-derievd exosomal miR-1246 induces M2 polarization of macrophages and reshapes the TME through increase the expression of IL-10, TGF $\beta$, and MMPs [161] (Fig. 3).

Abnormal differentiation and function of myeloid cells is a hallmark of cancer. Among them, myeloid-derived suppressor cells (MDSCs) have the function of suppressing adaptive immunity and innate immune response, and play an important role in tumor immune escape [162-164]. Exosomal miRNAs affect the function of
MDSCs by regulating the activity of transcription factors and transcription activators, thereby reshaping the immune microenvironment. In the research of glioblastoma, exosomal miR-10a targets RORA and affects the differentiation of MDSCs through the NFkB pathway, exosomal miR-21 targets PTEN and affects the activation of MDSCs via the p-STAT3/p-p65/p-Akt pathway [165]. Exosomal miR-155 istransmitted to monocytes, leading to nuclear translocation of NFkB and phosphorylation of STAT1, reprograming conventional monocytes into MDSCs [166]. Changes in the function of MDSCs affect the progression of the tumor itself. Recent research shows that exosmal miR-126a derived from MDSCs promotes angiogenesis and benefit breast cancer lung metastases [167].

The immunomodulation induced by exosomal miRNAs is complex and dynamic. In TME, tumor cells interact with various types of immune cells and crosspromote immunosuppressive activity. Among them, exosomal miRNAs play a pivotal role in them, but the mechanism has not been elucidated. Thereby, the function of exosomal miRNAs in the reciprocal interplays between cancer cells and hosts immune system merits further investigation.

\section{Perspectives of exosomal miRNAs}

With the vigorous development of the biology of exosomes in tumors, more and more evidence indicates that exosomal miRNAs play an important role in tumor progression and TME reshaping. Compared with miRNAs released directly into the circulatory system, exosomal miRNAs are protected by lipid bilayer encapsulation and avoid degradation by ribonuclease in the blood. Notebalely, exosomal miRNAs are more bioactive pool of circulating miRNAs compared to those miRNAs transported with liposomes [41, 168, 169]. Considering the advantages of exosomal miRNAs and the widespread presence of exosomes in all biological fluids (blood, breast milk, semen, and urine), diagnostic and therapeutic technologies based on exosomal miRNAs have a bright future.

Some specific exosomal miRNAs have high diagnostic value in tumors, and detecting them is helpful for early diagnosis of tumors. For example, in prostate cancer, breast cancer, and oral squamous cell carcinoma, the expression of exosomal miR-1246 is closely related to pathological grades, distant metastasis and poor prognosis [170-173]. Circulating exosomal miR-375 is valuable for the diagnosis of ovarian, rectal and prostate cancer [174-176]. The combination of multiple exosomal ncRNAs can enhance the diagnostic and prognostic potential of exosomal miRNAs. For example, the combination of expression of plasma exosomal miR-30d-5p and let-7d-3p is valuable diagnostic markers for non-invasive screening of cervical cancer and its precursors [177]. 


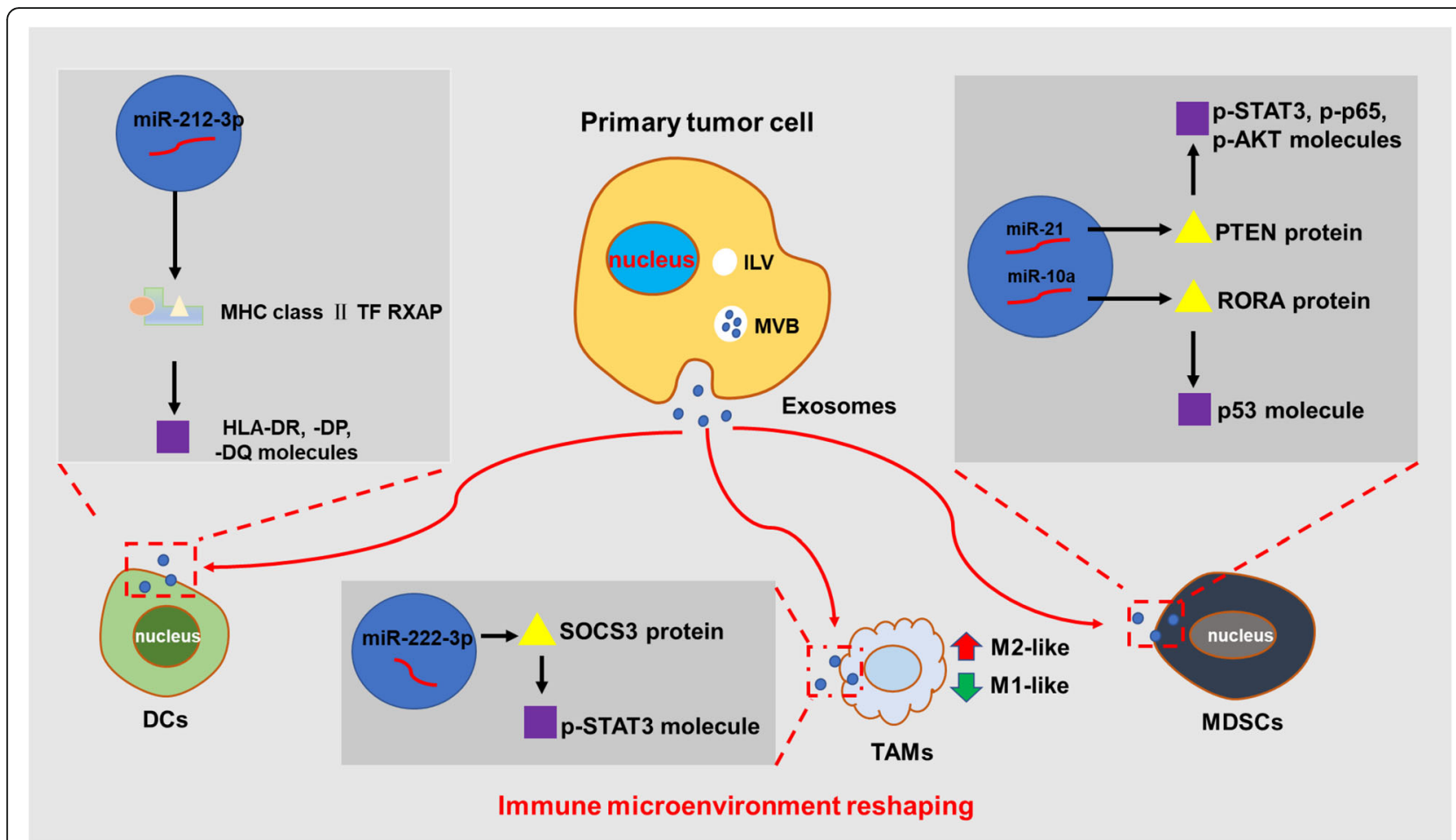

Fig. 3. The mechanism of immune microenvironment reshaping by exosomal miRNAs. Exosomes secreted by the primary tumor cells are taken up by the receptor immune cells, wherein the exosomal miR-212-3p target the MHC class II TF RFXAP complexus and activate the HLA-DR, -DP, and -DQ molecules. The exosomal miRNAs (miR-222-3p, miR-21, miR-10a, etc.) target the proteins (SOCS3, PTEN, RORA, etc.) and activate the molecules ( $\mathrm{p}$-STAT3, p-p65, p-AKT, p53, etc.). These exosomal miRNAs reshape inmune microenvironment by mediating immunosuppression.

Circulating exosomal miRNA-21 and lncRNA-ATB are related to the TNM stage of liver cancer and other prognostic factors, including the $\mathrm{T}$ stage and portal vein thrombosis [178].

Exosomal miRNAs, as a new tumor treatment method, are being widely explored. Based on the fact that exosomal miRNAs effectively bind to target mRNA and inhibit gene expression in recipient cells, related exosomal engineering techniques have been used to treat tumors by delivering tumor suppressor exosomal miRNAs. For example, delivery of exogenous miR-155 into DCs using TEX as a vector results in increased expression of MHCII (I/A-I/E), CD86, CD40 and CD83, promoting activation of DCs. Exosomal miRNA-155 significantly increases the levels of IL12p70, IFN- $\gamma$ and IL10 and improves immune function [179]. By fusing Her2 affinity to the extracellular $\mathrm{N}$-terminus of human Lamp2, and then using the modified exosomes to co-deliver 5-FU and miR-21 inhibitors (miR-21i), which targets colon cancer cells, effectively reverses the resistance of tumor cells and significantly enhances the toxicity of 5-FU resistant cancer cells [180].

Although exosomal miRNAs have made exciting progress in oncology, most of these results are experimental. Extension of exosomal miRNAs technologies to clinic remains challenging. There is no doubt that the function of exosomes is determined by their specific contents. A large amount of literature has reported that tumor-derived exosomal miRNAs can reshape TME and promote tumor progression, but little is known about the sorting mechanism of exosomal miRNAs. Although the basic framework of the endosome sorting complex required for transport (ESCRT) and Ago2 in MVB sorting has been reported in previous studies, it remains to be elucidate whether other novel sorting signals are involved in the release of exosomal miRNAs [86, 181-184].

The potential of exosomal miRNAs as diagnostic markers is unquestionable, but how to improve the sensitivity and specificity of exosomal miRNAs remains to be solved. The combination of different exosomal cargos, such as proteins, lipids, RNA and miRNAs for cancer diagnosis and prognosis can more comprehensively reflect the characteristics of tumors. At the same time, the scope of application of exosomal miRNAs also needs attention. The expression level of exosomal miRNAs is related to tumor types, clinical stages or other underlying diseases, and there are differences between individual patients. Therefore, how to use exosomal miRNAs accurately is also worth of considering.

The widespread use of exosomal miRNAs in clinical treatment remains challenging. First, exosomes-based therapeutic tools require more accurate and 
standardized exosomal purification methods, and the economic cost of mass-producing exsomes for clinical application cannot be ignored $[56,57]$. The second is that exosomal miRNAs-induced biological behavioral changes are often released through the cultivation of supra-physiological numbers of cell, and how many orders of magnitude of exosomal miRNAs are needed to achieve the corresponding efficacy in clinical applications remains to be determined.

\section{Conclusion}

Exosomal miRNAs, as a signaling molecule for communication between tumor cells and TME, play an important role in the formation and remodeling of TME, but its regulatory mechanism is still worth of further exploration. At present, most of the biological studies of exosomal miRNAs have been revealed by cell-culture systems in vitro. But the problems still remain whether exosomal miRNAs derived from supra-physiological numbers of cell reflect the biological conditions in vivo. It is necessary to conduct more experiments in vivo or in mammals.

With the increase of exosomes researches, people have gradually discovered that the exosomes obtained by traditional exosomal separation and purification methods (ultracentrifugation, density-gradient centrifugation, immune-affinity capture, and precipitation) not only contain SEVs, but also contain non-membrane structure vesicles (NVs). Components, double-stranded DNA (dsDNA) and histones, are more in the NVs rather than in exosomes or sEVs. Moreover, many of the most abundant miRNAs were more associated with extracellular $\mathrm{NV}$ fractions than with either parental cells or sEV fractions [56]. This indicates that we may need to reevaluate the composition of exosomes, and it is urgent to explore the generation and sorting mechanisms of exosomal miRNAs or miRNAs in other type of sEVs.

\footnotetext{
Abbreviations

TME: Tumor microenvironment; CAFs: Cancer-associated fibroblasts; ECM: Extracellular matrix; MMPs: Matrix metalloproteinases; HIF-1a: Hypoxia inducible factor-1a; VEGF: Vascular endothelial growth factor; miRNAs: MicroRNAs; NFs: Normal fibroblasts; EVs: Extracellular vesicles; sEVs: Small extracellular vesicle; ILV: Intraluminal vesicles; MVB: Multivesicular bodies; nSMase2: Neural Sphingomyelinase 2; hnRNP: Heterogeneous nuclear Ribonucleoprotein; Ago2: Argonaute 2; a-SMA: a-Smooth muscle actin; FGF2: Fibroblast growth factor 2; FAP: Fibroblast activating protein; SPHK1: Sphingosine kinase 1; CRC: Colorectal cancer; HCC: Hepatocarcinoma; NPC: Nasopharyngeal carcinoma; HUVECs: Human umbilical vein endothelial cells; MSCs: Mesenchymal stem cells; PHD1 and PHD2: Prolyl hydroxylase 1 and 2; HLSCs: Human liver stem-like cells; DCs: Dendritic cells; NKs: Natural killer cells; Treg: Regulatory T cells; TAMs: Tumor-associated macrophages; EOC: Epithelial ovarian cancer; MDSCs: Myeloid-derived suppressor cells; ESCRT: Endosome sorting complex required for transport; NVs: Nonmembrane structure vesicles; dsDNA: Double-stranded DNA; GC: Gastric cancer; NCSLC: Non-small cell lung cancer; CLL: Chronic lymphocytic leukemia.
}

\section{Authors' contributions}

Shiming Tan and Longzheng Xia contributed to drafting and editing of the manuscript, shared the first authorship. Qianjin Liao and Yujuan Zhou designed, revised and finalized the manuscript. Pin Yi, Qing Pan, Lu Tang, Yaqian Han, Jiaxin Liang, Linda Oyang, and Deliang Cao participated in the drafting and editing manuscript. Yutong Tian, Shan Rao participated in the revision and coordination. Pin Yi, Jinguan Lin, Shiming Tan contributed to literature search. Min Su, Yingrui Shi participated in the conception and coordination. All authors contributed toward data analysis, drafting and revising the paper and agreed to be accountable for all aspects of the work. All authors read and approved the final manuscript.

\section{Funding}

This work was supported in part by grants from the following sources: the National Natural Science Foundation of China $(81972636,81872281)$, the Natural Science Foundation of Hunan Province (2019JJ40175, 2019JJ40183, 2018JJ1013, 2017JJ3190), the Research Project of the Health and Family Planning Commission of Hunan Province (B20180400, B20180582), and the Changsha Science and Technology Board (kq1706045, kq1706043), Ascend Foundation of National cancer center (NCC2018b68).

\section{Availability of data and materials \\ Not applicable.}

Ethics approval and consent to participate

Not applicable.

Consent for publication

Not applicable.

\section{Competing interests}

The authors declare that they have no competing interests.

\section{Author details}

${ }^{1}$ Hunan Key Laboratory of Translational Radiation Oncology, Hunan Cancer Hospital and The Affiliated Cancer Hospital of Xiangya School of Medicine, Central South University, 283 Tongzipo Road, Changsha 410013, Hunan, China. ${ }^{2}$ University of South China, Hengyang 421001, Hunan, China. ${ }^{3}$ Department of Medical Microbiology, Immunology \& Cell Biology, Simmons Cancer Institute, Southern Illinois University School of Medicine, $913 \mathrm{~N}$. Rutledge Street, Springfield IL 62794, USA.

Received: 10 January 2020 Accepted: 6 April 2020

Published online: 16 April 2020

\section{References}

1. Li K, Chen Y, Li A, Tan C, Liu X. Exosomes play roles in sequential processes of tumor metastasis. Int J Cancer. 2019;144(7):1486-95.

2. Giraldo NA, Sanchez-Salas R, Peske JD, Vano Y, Becht E, Petitprez F, et al. The clinical role of the TME in solid cancer. Br J Cancer. 2019;120(1):45-53.

3. Rossi GR, Trindade ES, Souza-Fonseca-Guimaraes F. Tumor Microenvironment-Associated Extracellular Matrix Components Regulate NK Cell Function. Front Immunol. 2020;11:73.

4. Zeltz C, Primac I, Erusappan P, Alam J, Noel A, Gullberg D. Cancer-associated fibroblasts in desmoplastic tumors: emerging role of integrins. Semin Cancer Biol. 2020;62:166-81.

5. Hanahan D, Coussens LM. Accessories to the crime: functions of cells recruited to the tumor microenvironment. Cancer Cell. 2012;21(3):309-22.

6. Ma L, Hernandez MO, Zhao Y, Mehta M, Tran B, Kelly M, et al. Tumor Cell Biodiversity Drives Microenvironmental Reprogramming in Liver Cancer. Cancer Cell. 2019;36(4):418-30 e6.

7. Zhang X, Shen L, Liu Q, Hou L, Huang L. Inhibiting PI3 kinase-gamma in both myeloid and plasma cells remodels the suppressive tumor microenvironment in desmoplastic tumors. J Control Release. 2019;309:173-80.

8. Clement-Colmou K, Potiron V, Pietri M, Guillonneau M, Jouglar E, Chiavassa S, et al. Influence of Radiotherapy Fractionation Schedule on the Tumor Vascular Microenvironment in Prostate and Lung Cancer Models. Cancers (Basel). 2020;12:1.

9. Petrova V, Annicchiarico-Petruzzelli M, Melino G, Amelio I. The hypoxic tumour microenvironment. Oncogenesis. 2018;7(1):10. 
10. Armignacco R, Cantini G, Poli G, Guasti D, Nesi G, Romagnoli P, et al. The Adipose Stem Cell as a Novel Metabolic Actor in Adrenocortical Carcinoma Progression: Evidence from an In Vitro Tumor Microenvironment Crosstalk Model. Cancers (Basel). 2019;11:12.

11. Lei $X$, Lei $Y$, Li JK, Du WX, Li RG, Yang J, et al. Immune cells within the tumor microenvironment: Biological functions and roles in cancer immunotherapy. Cancer Lett. 2020;470:126-33.

12. Zhang H, Deng T, Liu R, Ning T, Yang H, Liu D, et al. CAF secreted miR-522 suppresses ferroptosis and promotes acquired chemo-resistance in gastric cancer. Mol Cancer. 2020;19(1):43.

13. De Palma M, Biziato D, Petrova TV. Microenvironmental regulation of tumour angiogenesis. Nat Rev Cancer. 2017;17(8):457-74.

14. Moriwaki K, Asahi M. Augmented TME O-GlcNAcylation Promotes Tumor Proliferation through the Inhibition of p38 MAPK. Mol Cancer Res. 2017; 15(9):1287-98.

15. Anfossi S, Fu X, Nagvekar R, Calin GA. MicroRNAs, Regulatory Messengers Inside and Outside Cancer Cells. Adv Exp Med Biol. 2018;1056:87-108.

16. Fattore L, Ruggiero CF, Pisanu ME, Liguoro D, Cerri A, Costantini S, et al. Reprogramming miRNAs global expression orchestrates development of drug resistance in BRAF mutated melanoma. Cell Death Differ. 2019;26(7): 1267-82.

17. Malhotra A, Sharma U, Puhan S, Chandra Bandari N, Kharb A, Arifa PP, et al. Stabilization of miRNAs in esophageal cancer contributes to radioresistance and limits efficacy of therapy. Biochimie. 2019;156:148-57.

18. Yan W, Wu X, Zhou W, Fong MY, Cao M, Liu J, et al. Cancer-cell-secreted exosomal miR-105 promotes tumour growth through the MYC-dependent metabolic reprogramming of stromal cells. Nat Cell Biol. 2018;20(5):597-609.

19. Zhou CF, Ma J, Huang L, Yi HY, Zhang YM, Wu XG, et al. Cervical squamous cell carcinoma-secreted exosomal miR-221-3p promotes lymphangiogenesis and lymphatic metastasis by targeting VASH1. Oncogene. 2019;38(8):1256-68.

20. Cai L, Ye Y, Jiang Q, Chen Y, Lyu X, Li J, et al. Epstein-Barr virus-encoded microRNA BART1 induces tumour metastasis by regulating PTEN-dependent pathways in nasopharyngeal carcinoma. Nat Commun. 2015;6:7353.

21. Tian R, Wang J, Yan H, Wu J, Xu Q, Zhan X, et al. Differential expression of miR16 in glioblastoma and glioblastoma stem cells: their correlation with proliferation, differentiation, metastasis and prognosis. Oncogene. 2017; 36(42):5861-73.

22. Xiao X, Lu Z, Lin V, May A, Shaw DH, Wang Z, et al. MicroRNA miR-24-3p Reduces Apoptosis and Regulates Keap1-Nrf2 Pathway in Mouse Cardiomyocytes Responding to Ischemia/Reperfusion Injury. Oxid Med Cell Longev. 2018;2018:7042105.

23. Song J, Ma Q, Hu M, Qian D, Wang B, He N. The Inhibition of miR-144-3p on Cell Proliferation and Metastasis by Targeting TOP2A in HCMV-Positive Glioblastoma Cells. Molecules. 2018;23:12.

24. Sun J, Hong J, Sun S, Wang X, Peng Y, Zhou J, et al. Transcription factor 7like 2 controls matrix degradation through nuclear factor kappaB signaling and is repressed by microRNA-155 in nucleus pulposus cells. Biomed Pharmacother. 2018;108:646-55.

25. Uddin MN, Li M, Wang X. Identification of Transcriptional Markers and microRNAmRNA Regulatory Networks in Colon Cancer by Integrative Analysis of mRNA and microRNA Expression Profiles in Colon Tumor Stroma. Cells. 2019;8:9.

26. Slack FJ, Chinnaiyan AM. The Role of Non-coding RNAs in Oncology. Cell. 2019;179(5):1033-55.

27. Kanchan RK, Siddiqui JA, Mahapatra S, Batra SK, Nasser MW. microRNAs Orchestrate Pathophysiology of Breast Cancer Brain Metastasis: Advances in Therapy. Mol Cancer. 2020;19(1):29.

28. Conti I, Varano G, Simioni C, Laface I, Milani D, Rimondi E, et al. miRNAs as Influencers of Cell-Cell Communication in Tumor Microenvironment. Cells. 2020;9:1.

29. Tang X, Hou Y, Yang G, Wang X, Tang S, Du YE, et al. Stromal miR-200s contribute to breast cancer cell invasion through CAF activation and ECM remodeling. Cell Death Differ. 2016;23(1):132-45.

30. Baroni S, Romero-Cordoba S, Plantamura I, Dugo M, D'Ippolito E, Cataldo A, et al. Exosome-mediated delivery of miR-9 induces cancer-associated fibroblast-like properties in human breast fibroblasts. Cell Death Dis. 2016;7(7):e2312.

31. Hunter S, Nault B, Ugwuagbo KC, Maiti S, Majumder M. Mir526b and Mir655 Promote Tumour Associated Angiogenesis and Lymphangiogenesis in Breast Cancer. Cancers (Basel). 2019;11:7

32. Liu Y, Li X, Zhang Y, Wang H, Rong X, Peng J, et al. An miR-340-5pmacrophage feedback loop modulates the progression and tumor microenvironment of glioblastoma multiforme. Oncogene. 2019;38(49): 7399-415.
33. Chen EB, Zhou ZJ, Xiao K, Zhu GQ, Yang Y, Wang B, et al. The miR-561-5p/ CX3CL1 Signaling Axis Regulates Pulmonary Metastasis in Hepatocellular Carcinoma Involving CX3CR1(+) Natural Killer Cells Infiltration. Theranostics. 2019:9(16):4779-94.

34. Wu F, Li F, Lin X, Xu F, Cui R. Zhong J, et al. Endocr Relat Cancer: Exosomes increased angiogenesis in papillary thyroid cancer microenvironment; 2019.

35. Hwang WL, Lan HY, Cheng WC, Huang SC, Yang MH. Tumor stem-like cellderived exosomal RNAs prime neutrophils for facilitating tumorigenesis of colon cancer. J Hematol Oncol. 2019;12(1):10.

36. Kalluri R, LeBleu VS. The biology, function, and biomedical applications of exosomes. Science. 2020;367:6478.

37. Tian X, Shen H, Li Z, Wang T, Wang S. Tumor-derived exosomes, myeloidderived suppressor cells, and tumor microenvironment. J Hematol Oncol. 2019;12(1):84

38. Whiteside TL. Exosome and mesenchymal stem cell cross-talk in the tumor microenvironment. Semin Immunol. 2018;35:69-79.

39. Cheng WC, Liao TT, Lin CC, Yuan LE, Lan HY, Lin HH, et al. RAB27B-activated secretion of stem-like tumor exosomes delivers the biomarker microRNA-146a$5 p$, which promotes tumorigenesis and associates with an immunosuppressive tumor microenvironment in colorectal cancer. Int J Cancer. 2019.

40. Patel H, Nilendu P, Jahagirdar D, Pal JK, Sharma NK. Modulating secreted components of tumor microenvironment: $A$ masterstroke in tumor therapeutics. Cancer Biol Ther. 2018;19(1):3-12.

41. Wang M, Yu F, Ding H, Wang Y, Li P, Wang K. Emerging Function and Clinical Values of Exosomal MicroRNAs in Cancer. Mol Ther Nucleic Acids. 2019;16:791-804.

42. Sun Z, Shi K, Yang S, Liu J, Zhou Q, Wang G, et al. Effect of exosomal miRNA on cancer biology and clinical applications. Mol Cancer. 2018;17(1):147.

43. Pontecorvi G, Bellenghi M, Puglisi R, Care A, Mattia G. Tumor-derived extracellular vesicles and microRNAs: Functional roles, diagnostic, prognostic and therapeutic options. Cytokine Growth Factor Rev. 2020;51:75-83.

44. Lucero R, Zappulli V, Sammarco A, Murillo OD, Cheah PS, Srinivasan S, et al. Glioma-Derived miRNA-Containing Extracellular Vesicles Induce Angiogenesis by Reprogramming Brain Endothelial Cells. Cell Rep. 2020;30(7):2065-74 e4.

45. Varricchi G, Loffredo S, Galdiero MR, Marone G, Cristinziano L, Granata F, et al. Innate effector cells in angiogenesis and lymphangiogenesis. Curr Opin Immunol. 2018;53:152-60.

46. Xie F, Zhou X, Fang M, Li H, Su P, Tu Y, et al. Extracellular Vesicles in Cancer Immune Microenvironment and Cancer Immunotherapy. Adv Sci (Weinh). 2019;6(24):1901779.

47. Daassi D, Mahoney KM, Freeman GJ. The importance of exosomal PDL1 in tumour immune evasion. Nat Rev Immunol. 2020;20(4):209-15.

48. Jarosz-Biej M, Smolarczyk R, Cichon T, Kulach N. Tumor Microenvironment as A "Game Changer" in Cancer Radiotherapy. Int J Mol Sci. 2019;20:13.

49. Peng J, Yang Q, Shi K, Xiao Y, Wei X, Qian Z. Intratumoral fate of functional nanoparticles in response to microenvironment factor: Implications on cancer diagnosis and therapy. Adv Drug Deliv Rev. 2019;143:37-67.

50. Gao X, Zhang Z, Mashimo T, Shen B, Nyagilo J, Wang H, et al. Gliomas Interact with Non-glioma Brain Cells via Extracellular Vesicles. Cell Rep. 2020; 30(8):2489-500 e5

51. van Niel G, D'Angelo G, Raposo G. Shedding light on the cell biology of extracellular vesicles. Nat Rev Mol Cell Biol. 2018;19(4):213-28.

52. Yekula A, Yekula A, Muralidharan K, Kang K, Carter BS, Balaj L. Extracellular Vesicles in Glioblastoma Tumor Microenvironment. Front Immunol. 2019;10:3137.

53. Xie $M$, Xiong $W$, She $Z$, Wen $Z$, Abdirahman AS, Wan W, et al. Immunoregulatory Effects of Stem Cell-Derived Extracellular Vesicles on Immune Cells. Front Immunol. 2020;11:13.

54. Harmati M, Gyukity-Sebestyen E, Dobra G, Janovak L, Dekany I, Saydam O, et al. Small extracellular vesicles convey the stress-induced adaptive responses of melanoma cells. Sci Rep. 2019;9(1):15329.

55. Thery C, Witwer KW, Aikawa E, Alcaraz MJ, Anderson JD, Andriantsitohaina R, et al. Minimal information for studies of extracellular vesicles 2018 (MISEV2018): a position statement of the International Society for Extracellular Vesicles and update of the MISEV2014 guidelines. J Extracell Vesicles. 2018;7(1):1535750

56. Jeppesen DK, Fenix AM, Franklin JL, Higginbotham JN, Zhang Q, Zimmerman LJ, et al. Reassessment of Exosome Composition. Cell. 2019; 177(2):428-45 e18

57. Tian Y, Gong M, Hu Y, Liu H, Zhang W, Zhang M, et al. Quality and efficiency assessment of six extracellular vesicle isolation methods by nanoflow cytometry. J Extracell Vesicles. 2020;9(1):1697028. 
58. Kalluri R. The biology and function of exosomes in cancer. J Clin Invest. 2016;126(4):1208-15

59. Mathieu M, Martin-Jaular L, Lavieu G, Thery C. Specificities of secretion and uptake of exosomes and other extracellular vesicles for cell-to-cell communication. Nat Cell Biol. 2019;21(1):9-17.

60. Bebelman MP, Smit MJ, Pegtel DM, Baglio SR. Biogenesis and function of extracellular vesicles in cancer. Pharmacol Ther. 2018;188:1-11.

61. Rajagopal C, Harikumar KB. The Origin and Functions of Exosomes in Cancer. Front Oncol. 2018;8:66.

62. Kowal J, Tkach M, Thery C. Biogenesis and secretion of exosomes. Curr Opin Cell Biol. 2014;29:116-25.

63. Hyenne V, Labouesse M, Goetz JG. The Small GTPase Ral orchestrates MVB biogenesis and exosome secretion. Small GTPases. 2018;9(6):445-51.

64. Mills JT, Schwenzer A, Marsh EK, Edwards MR, Sabroe I, Midwood KS, et al. Airway Epithelial Cells Generate Pro-inflammatory Tenascin-C and Small Extracellular Vesicles in Response to TLR3 Stimuli and Rhinovirus Infection. Front Immunol. 2019;10:1987.

65. Gao K, Jin J, Huang C, Li J, Luo H, Li L, et al. Exosomes Derived From Septic Mouse Serum Modulate Immune Responses via Exosome-Associated Cytokines. Front Immunol. 2019;10:1560.

66. Castano C, Kalko S, Novials A, Parrizas M. Obesity-associated exosomal miRNAs modulate glucose and lipid metabolism in mice. Proc Natl Acad Sci U S A. 2018;115(48):12158-63.

67. Zhang Y, Hu YW, Zheng L, Wang Q. Characteristics and Roles of Exosomes in Cardiovascular Disease. DNA Cell Biol. 2017;36(3):202-11.

68. Gao G, Li C, Zhu J, Wang Y, Huang Y, Zhao S, et al. Glutaminase 1 Regulates Neuroinflammation After Cerebral Ischemia Through Enhancing Microglial Activation and Pro-Inflammatory Exosome Release. Front Immunol. 2020;11: 161.

69. Lai N, Wu D, Liang T, Pan P, Yuan G, Li X, et al. Systemic exosomal miR193b-3p delivery attenuates neuroinflammation in early brain injury after subarachnoid hemorrhage in mice. J Neuroinflammation. 2020;17(1):74.

70. Ge X, Guo M, Hu T, Li W, Huang S, Yin Z, et al. Increased Microglial Exosomal miR-124-3p Alleviates Neurodegeneration and Improves Cognitive Outcome after rmTBI. Mol Ther. 2020;28(2):503-22.

71. Gerloff D, Lutzkendorf J, Moritz RKC, Wersig T, Mader K, Muller LP, et al. Melanoma-Derived Exosomal miR-125b-5p Educates Tumor Associated Macrophages (TAMs) by Targeting Lysosomal Acid Lipase A (LIPA). Cancers (Basel). 2020;12:2.

72. Xiao Y, Zhong J, Zhong B, Huang J, Jiang L, Jiang Y, et al. Exosomes as potential sources of biomarkers in colorectal cancer. Cancer Lett. 2020;476: $13-22$.

73. Castellanos-Rizaldos E, Grimm DG, Tadigotla V, Hurley J, Healy J, Neal PL, et al. Exosome-Based Detection of EGFR T790M in Plasma from Non-Small Cell Lung Cancer Patients. Clin Cancer Res. 2018;24(12):2944-50.

74. Li ZG, Scott MJ, Brzoska T, Sundd P, Li YH, Billiar TR, et al. Lung epithelial cell-derived IL-25 negatively regulates LPS-induced exosome release from macrophages. Mil Med Res. 2018;5(1):24.

75. Zhang Z, Xing T, Chen Y, Xiao J. Exosome-mediated miR-200b promotes colorectal cancer proliferation upon TGF-beta1 exposure. Biomed Pharmacother. 2018;106:1135-43.

76. Wu CX, Liu ZF. Proteomic Profiling of Sweat Exosome Suggests its Involvement in Skin Immunity. J Invest Dermatol. 2018;138(1):89-97.

77. Zhang P, Zhou H, Lu K, Lu Y, Wang Y, Feng T. Exosome-mediated delivery of MALAT1 induces cell proliferation in breast cancer. Onco Targets Ther. 2018;11:291-9.

78. Guay C, Regazzi R. Exosomes as new players in metabolic organ cross-talk. Diabetes Obes Metab. 2017;19(Suppl 1):137-46.

79. Schwarzenbach H, Gahan PB. MicroRNA Shuttle from Cell-To-Cell by Exosomes and Its Impact in Cancer. Noncoding RNA. 2019;5:1.

80. Kosaka N, Iguchi H, Hagiwara K, Yoshioka Y, Takeshita F, Ochiya T. Neutral sphingomyelinase 2 (nSMase2)-dependent exosomal transfer of angiogenic microRNAs regulate cancer cell metastasis. J Biol Chem. 2013;288(15):10849-59.

81. Santangelo L, Giurato G, Cicchini C, Montaldo C, Mancone C, Tarallo R, et al. The RNA-Binding Protein SYNCRIP Is a Component of the Hepatocyte Exosomal Machinery Controlling MicroRNA Sorting. Cell Rep. 2016;17(3): 799-808.

82. Villarroya-Beltri C, Gutierrez-Vazquez C, Sanchez-Cabo F, Perez-Hernandez D, Vazquez J, Martin-Cofreces N, et al. Sumoylated hnRNPA2B1 controls the sorting of miRNAs into exosomes through binding to specific motifs. Nat Commun. 2013;4:2980.
83. Koppers-Lalic D, Hackenberg M, Bijnsdorp IV, van Eijndhoven MAJ, Sadek P, Sie $D$, et al. Nontemplated nucleotide additions distinguish the small RNA composition in cells from exosomes. Cell Rep. 2014;8(6):1649-58.

84. Yokoi A, Villar-Prados A, Oliphint PA, Zhang J, Song X, De Hoff P, et al. Mechanisms of nuclear content loading to exosomes. Sci Adv. 2019;5(11): eaax8849.

85. Guduric-Fuchs J, O'Connor A, Camp B, O'Neill CL, Medina RJ, Simpson DA. Selective extracellular vesicle-mediated export of an overlapping set of microRNAs from multiple cell types. BMC Genomics. 2012;13:357.

86. McKenzie AJ, Hoshino D, Hong NH, Cha DJ, Franklin JL, Coffey RJ, et al. KRAS-MEK Signaling Controls Ago2 Sorting into Exosomes. Cell Rep. 2016; 15(5):978-87.

87. Xu R, Rai A, Chen M, Suwakulsiri W, Greening DW, Simpson RJ. Extracellular vesicles in cancer - implications for future improvements in cancer care. Nat Rev Clin Oncol. 2018;15(10):617-38.

88. Fabbri M. MicroRNAs and miRceptors: a new mechanism of action for intercellular communication. Philos Trans R Soc Lond B Biol Sci. 2018;373: 1737.

89. Li I, Nabet BY. Exosomes in the tumor microenvironment as mediators of cancer therapy resistance. Mol Cancer. 2019;18(1):32.

90. Wu P, Zhang B, Shi H, Qian H, Xu W. MSC-exosome: A novel cell-free therapy for cutaneous regeneration. Cytotherapy. 2018;20(3):291-301.

91. Zhan C, Yang X. Yin X, Hou J. Oral Dis: Exosomes and other extracellular vesicles in oral and salivary gland cancers; 2019.

92. Salarian $\mathrm{M}$, Ibhagui OY, Yang JJ. Molecular imaging of extracellular matrix proteins with targeted probes using magnetic resonance imaging. Wiley Interdiscip Rev Nanomed Nanobiotechnol; 2020. p. e1622.

93. Abyaneh HS, Regenold M, McKee TD, Allen C, Gauthier MA. Towards extracellular matrix normalization for improved treatment of solid tumors. Theranostics. 2020;10(4):1960-80.

94. Sun Y, Wang R, Qiao M, Xu Y, Guan W, Wang L. Cancer associated fibroblasts tailored tumor microenvironment of therapy resistance in gastrointestinal cancers. J Cell Physiol. 2018;233(9):6359-69.

95. Houthuiizen JM, Jonkers J. Cancer-associated fibroblasts as key regulators of the breast cancer tumor microenvironment. Cancer Metastasis Rev. 2018; 37(4):577-97.

96. Alkasalias T, Moyano-Galceran L, Arsenian-Henriksson M, Lehti K. Fibroblasts in the Tumor Microenvironment: Shield or Spear? Int J Mol Sci. 2018;19:5.

97. Karakasheva TA, Lin EW, Tang Q, Qiao E, Waldron TJ, Soni M, et al. IL-6 Mediates Cross-Talk between Tumor Cells and Activated Fibroblasts in the Tumor Microenvironment. Cancer Res. 2018;78(17):4957-70.

98. Webber J, Steadman R, Mason MD, Tabi Z, Clayton A. Cancer exosomes trigger fibroblast to myofibroblast differentiation. Cancer Res. 2010;70(23): 9621-30.

99. Wu HJ, Hao M, Yeo SK, Guan JL. FAK signaling in cancer-associated fibroblasts promotes breast cancer cell migration and metastasis by exosomal miRNAs-mediated intercellular communication. Oncogene. 2020.

100. Yang X, Li Y, Zou L, Zhu Z. Role of Exosomes in Crosstalk Between CancerAssociated Fibroblasts and Cancer Cells. Front Oncol. 2019;9:356.

101. Zhang $Y$, Cai H, Chen S, Sun D, Zhang D, He Y. Exosomal transfer of miR124 inhibits normal fibroblasts to cancer-associated fibroblasts transition by targeting sphingosine kinase 1 in ovarian cancer. J Cell Biochem. 2019.

102. Lu Y, Zhang T, Shan S, Wang S, Bian W, Ren T, et al. MiR-124 regulates transforming growth factor-beta1 induced differentiation of lung resident mesenchymal stem cells to myofibroblast by repressing Wnt/beta-catenin signaling. Dev Biol. 2019;449(2):115-21.

103. Frassanito MA, Desantis V, Di Marzo L, Craparotta I, Beltrame L, Marchini S, et al. Bone marrow fibroblasts overexpress miR-27b and miR-214 in step with multiple myeloma progression, dependent on tumour cell-derived exosomes. J Pathol. 2019;247(2):241-53.

104. Lv X, Li J, Hu Y, Wang S, Yang C, Li C, et al. Overexpression of miR-27b-3p Targeting Wnt3a Regulates the Signaling Pathway of Wnt/beta-Catenin and Attenuates Atrial Fibrosis in Rats with Atrial Fibrillation. Oxid Med Cell Longev. 2019;2019:5703764.

105. Wang J, Guan X, Zhang Y, Ge S, Zhang L, Li H, et al. Exosomal miR-27a Derived from Gastric Cancer Cells Regulates the Transformation of Fibroblasts into Cancer-Associated Fibroblasts. Cell Physiol Biochem. 2018; 49(3):869-83.

106. Dai G, Yao X, Zhang Y, Gu J, Geng Y, Xue F, et al. Colorectal cancer cellderived exosomes containing miR-10b regulate fibroblast cells via the PI3K/ Akt pathway. Bull Cancer. 2018;105(4):336-49. 
107. Liang HX, Sun LB, Liu NJ. Neferine inhibits proliferation, migration and invasion of U251 glioma cells by down-regulation of miR-10b. Biomed Pharmacother. 2019;109:1032-40.

108. Yoshii S, Hayashi Y, lijima H, Inoue T, Kimura K, Sakatani A, et al. Exosomal microRNAs derived from colon cancer cells promote tumor progression by suppressing fibroblast TP53 expression. Cancer Sci. 2019;110(8):2396-407.

109. Zhou X, Yan T, Huang C, Xu Z, Wang L, Jiang E, et al. Melanoma cellsecreted exosomal miR-155-5p induce proangiogenic switch of cancerassociated fibroblasts via SOCS1/JAK2/STAT3 signaling pathway. J Exp Clin Cancer Res. 2018:37(1):242.

110. Hu J, Huang CX, Rao PP, Cao GQ, Zhang Y, Zhou JP, et al. MicroRNA-155 inhibition attenuates endoplasmic reticulum stress-induced cardiomyocyte apoptosis following myocardial infarction via reducing macrophage inflammation. Eur J Pharmacol. 2019;857:172449.

111. Zhou Y, Ren H, Dai B, Li J, Shang L, Huang J, et al. Hepatocellular carcinoma-derived exosomal miRNA-21 contributes to tumor progression by converting hepatocyte stellate cells to cancer-associated fibroblasts. J Exp Clin Cancer Res. 2018;37(1):324.

112. Jiang C, Guo Y, Yu H, Lu S, Meng L. Pleiotropic microRNA-21 in pulmonary remodeling: novel insights for molecular mechanism and present advancements. Allergy Asthma Clin Immunol. 2019;15:33.

113. Fang T, Lv H, Lv G, Li T, Wang C, Han Q, et al. Tumor-derived exosomal miR1247-3p induces cancer-associated fibroblast activation to foster lung metastasis of liver cancer. Nat Commun. 2018;9(1):191.

114. Lawson J, Dickman C, Towle R, Jabalee J, Javer A, Garnis C. Extracellular vesicle secretion of miR-142-3p from lung adenocarcinoma cells induces tumor promoting changes in the stroma through cell-cell communication. Mol Carcinog. 2019;58(3):376-87.

115. Qin X, Guo H, Wang X, Zhu X, Yan M, Wang X, et al. Exosomal miR-196a derived from cancer-associated fibroblasts confers cisplatin resistance in head and neck cancer through targeting CDKN1B and ING5. Genome Biol. 2019;20(1):12.

116. Ham IH, Lee D, Hur H. Role of Cancer-Associated Fibroblast in Gastric Cancer Progression and Resistance to Treatments. J Oncol. 2019;2019: 6270784.

117. Deepak KGK, Vempati R, Nagaraju GP, Dasari VR, N S, Rao DN, et al. Tumor microenvironment: Challenges and opportunities in targeting metastasis of triple negative breast cancer. Pharmacol Res. 2020;153:104683.

118. Hashimoto K, Ochi H, Sunamura S, Kosaka N, Mabuchi Y, Fukuda T, et al. Cancer-secreted hsa-miR-940 induces an osteoblastic phenotype in the bone metastatic microenvironment via targeting ARHGAP1 and FAM134A. Proc Natl Acad Sci U S A. 2018;115(9):2204-9.

119. Wang FT, Sun W, Zhang JT, Fan YZ. Cancer-associated fibroblast regulation of tumor neo-angiogenesis as a therapeutic target in cancer. Oncol Lett. 2019;17(3):3055-65.

120. Zhou W, Fong MY, Min Y, Somlo G, Liu L, Palomares MR, et al. Cancersecreted miR-105 destroys vascular endothelial barriers to promote metastasis. Cancer Cell. 2014;25(4):501-15.

121. Bao L, You B, Shi S, Shan Y, Zhang Q, Yue H, et al. Metastasis-associated miR-23a from nasopharyngeal carcinoma-derived exosomes mediates angiogenesis by repressing a novel target gene TSGA10. Oncogene. 2018; 37(21):2873-89.

122. Lu S, Xu Q. MicroRNA-23a inhibits melanoma cell proliferation, migration, and invasion in mice through a negative feedback regulation of sdcbp and the MAPK/ERK signaling pathway. IUBMB Life. 2019;71(5):587-600.

123. Sun X, Ma X, Wang J, Zhao Y, Wang Y, Bihl JC, et al. Glioma stem cellsderived exosomes promote the angiogenic ability of endothelial cells through miR-21NEGF signal. Oncotarget. 2017;8(22):36137-48.

124. Sabry D, El-Deek SEM, Maher M, El-Baz MAH, El-Bader HM, Amer E, et al. Role of miRNA-210, miRNA-21 and miRNA-126 as diagnostic biomarkers in colorectal carcinoma: impact of HIF-1alpha-VEGF signaling pathway. Mol Cell Biochem. 2019;454(1-2):177-89.

125. Lin XJ, Fang JH, Yang XJ, Zhang C, Yuan Y, Zheng L, et al. Hepatocellular Carcinoma Cell-Secreted Exosomal MicroRNA-210 Promotes Angiogenesis In Vitro and In Vivo. Mol Ther Nucleic Acids. 2018;11:243-52.

126. Masoumi-Dehghi S, Babashah S, Sadeghizadeh M. microRNA-141-3pcontaining small extracellular vesicles derived from epithelial ovarian cancer cells promote endothelial cell angiogenesis through activating the JAK STAT3 and NF-kappaB signaling pathways. J Cell Commun Signal. 2020.

127. Pakravan K, Babashah S, Sadeghizadeh M, Mowla SJ, MossahebiMohammadi M, Ataei F, et al. MicroRNA-100 shuttled by mesenchymal stem cell-derived exosomes suppresses in vitro angiogenesis through modulating the mTOR/HIF-1alpha/NEGF signaling axis in breast cancer cells. Cell Oncol (Dordr). 2017:40(5):457-70.

128. Kim DH, Park S, Kim H, Choi YJ, Kim SY, Sung KJ, et al. Tumor-derived exosomal miR-619-5p promotes tumor angiogenesis and metastasis through the inhibition of RCAN1.4. Cancer Lett. 2020;475:2-13.

129. He L, Zhu W, Chen Q, Yuan Y, Wang Y, Wang J, et al. Ovarian cancer cellsecreted exosomal miR-205 promotes metastasis by inducing angiogenesis. Theranostics. 2019;9(26):8206-20.

130. Zeng Z, Li Y, Pan Y, Lan X, Song F, Sun J, et al. Cancer-derived exosomal miR-25-3p promotes pre-metastatic niche formation by inducing vascular permeability and angiogenesis. Nat Commun. 2018;9(1):5395.

131. Farrugia MK, Vanderbilt DB, Salkeni MA, Ruppert JM. Kruppel-like Pluripotency Factors as Modulators of Cancer Cell Therapeutic Responses. Cancer Res. 2016;76(7):1677-82.

132. Hsu YL, Hung JY, Chang WA, Lin YS, Pan YC, Tsai PH, et al. Hypoxic lung cancer-secreted exosomal miR-23a increased angiogenesis and vascular permeability by targeting prolyl hydroxylase and tight junction protein ZO1. Oncogene. 2017;36(34):4929-42.

133. Chen X, Yang F, Zhang T, Wang W, Xi W, Li Y, et al. MiR-9 promotes tumorigenesis and angiogenesis and is activated by MYC and OCT4 in human glioma. J Exp Clin Cancer Res. 2019;38(1):99.

134. Zhao S, Li J, Zhang G, Wang Q, Wu C, Zhang Q, et al. Exosomal miR-451a Functions as a Tumor Suppressor in Hepatocellular Carcinoma by Targeting LPIN1. Cell Physiol Biochem. 2019;53(1):19-35.

135. Lu J, Liu QH, Wang F, Tan JJ, Deng YQ, Peng XH, et al. Exosomal miR-9 inhibits angiogenesis by targeting MDK and regulating PDK/AKT pathway in nasopharyngeal carcinoma. J Exp Clin Cancer Res. 2018;37(1):147.

136. Lopatina T, Grange C, Fonsato V, Tapparo M, Brossa A, Fallo S, et al. Extracellular vesicles from human liver stem cells inhibit tumor angiogenesis. Int J Cancer. 2019;144(2):322-33.

137. Vignard V, Labbe M, Marec N, Andre-Gregoire G, Jouand N, Fonteneau JF, et al. MicroRNAs in Tumor Exosomes Drive Immune Escape in Melanoma. Cancer Immunol Res. 2020;8(2):255-67.

138. Schmittgen TD. Exosomal miRNA Cargo as Mediator of Immune Escape Mechanisms in Neuroblastoma. Cancer Res. 2019;79(7):1293-4.

139. Netea-Maier RT, Smit JWA, Netea MG. Metabolic changes in tumor cells and tumorassociated macrophages: A mutual relationship. Cancer Lett. 2018:413:102-9.

140. van Dalen FJ, van Stevendaal M, Fennemann FL, Verdoes M, llina O. Molecular Repolarisation of Tumour-Associated Macrophages. Molecules. 2018;24:1.

141. Fotaki G, Jin C, Ramachandran M, Kerzeli IK, Karlsson-Parra A, Yu D, et al. Pro-inflammatory allogeneic DCs promote activation of bystander immune cells and thereby license antigen-specific T-cell responses. Oncoimmunology. 2018;7(3):e1395126.

142. Diao J, Gu H, Tang M, Zhao J, Cattral MS. Tumor Dendritic Cells (DCs) Derived from Precursors of Conventional DCs Are Dispensable for Intratumor CTL Responses. J Immunol. 2018;201(4):1306-14.

143. Kikete S, Chu X, Wang L, Bian Y. Endogenous and tumour-derived microRNAs regulate cross-presentation in dendritic cells and consequently cytotoxic T cell function. Cytotechnology. 2016;68(6):2223-33.

144. Montecalvo A, Larregina AT, Shufesky WJ, Stolz DB, Sullivan ML, Karlsson JM, et al. Mechanism of transfer of functional microRNAs between mouse dendritic cells via exosomes. Blood. 2012;119(3):756-66.

145. Ding G, Zhou L, Shen T, Cao L. IFN-gamma induces the upregulation of RFXAP via inhibition of miR-212-3p in pancreatic cancer cells: A novel mechanism for IFN-gamma response. Oncol Lett. 2018;15(3):3760-5.

146. Ding G, Zhou L, Qian Y, Fu M, Chen J, Chen J, et al. Pancreatic cancerderived exosomes transfer miRNAs to dendritic cells and inhibit RFXAP expression via miR-212-3p. Oncotarget. 2015;6(30):29877-88.

147. Zhou M, Chen J, Zhou L, Chen W, Ding G, Cao L. Pancreatic cancer derived exosomes regulate the expression of TLR4 in dendritic cells via miR-203. Cell Immunol. 2014;292(1-2):65-9.

148. Taghikhani A, Hassan ZM, Ebrahimi M, Moazzeni SM. microRNA modified tumor-derived exosomes as novel tools for maturation of dendritic cells. J Cell Physiol. 2019;234(6):9417-27.

149. Tung SL, Boardman DA, Sen M, Letizia M, Peng Q, Cianci N, et al. Regulatory T cell-derived extracellular vesicles modify dendritic cell function. Sci Rep. 2018;8(1):6065.

150. Okoye IS, Coomes SM, Pelly VS, Czieso S, Papayannopoulos V, Tolmachova T, et al. MicroRNA-Containing T-Regulatory-Cell-Derived Exosomes Suppress Pathogenic T Helper 1 Cells. Immunity. 2014;41(3):503. 
151. Grossman JG, Nywening TM, Belt BA, Panni RZ, Krasnick BA, DeNardo DG, et al. Recruitment of CCR2(+) tumor associated macrophage to sites of liver metastasis confers a poor prognosis in human colorectal cancer. Oncoimmunology. 2018;7(9):e1470729.

152. Eichmuller SB, Osen W, Mandelboim O, Seliger B. Immune Modulatory microRNAs Involved in Tumor Attack and Tumor Immune Escape. J Natl Cancer Inst. 2017;109:10.

153. Choo YW, Kang M, Kim HY, Han J, Kang S, Lee JR, et al. M1 MacrophageDerived Nanovesicles Potentiate the Anticancer Efficacy of Immune Checkpoint Inhibitors. ACS Nano. 2018;12(9):8977-93.

154. Trivedi M, Talekar M, Shah P, Ouyang Q, Amiji M. Modification of tumor cell exosome content by transfection with wt-p53 and microRNA-125b expressing plasmid DNA and its effect on macrophage polarization. Oncogenesis. 2016;5(8):e250.

155. Hsieh CH, Tai SK, Yang MH. Snail-overexpressing Cancer Cells Promote M2Like Polarization of Tumor-Associated Macrophages by Delivering MiR-21Abundant Exosomes. Neoplasia. 2018;20(8):775-88.

156. Ying X, Wu Q, Wu X, Zhu Q, Wang X, Jiang L, et al. Epithelial ovarian cancersecreted exosomal miR-222-3p induces polarization of tumor-associated macrophages. Oncotarget. 2016;7(28):43076-87.

157. Chen $X$, Zhou J, Li X, Wang $X$, Lin Y, Wang X. Exosomes derived from hypoxic epithelial ovarian cancer cells deliver microRNAs to macrophages and elicit a tumor-promoted phenotype. Cancer Lett. 2018;435:80-91.

158. Chen X, Ying X, Wang X, Wu X, Zhu Q, Wang X. Exosomes derived from hypoxic epithelial ovarian cancer deliver microRNA-940 to induce macrophage M2 polarization. Oncol Rep. 2017;38(1):522-8.

159. Wang X, Luo G, Zhang K, Cao J, Huang C, Jiang T, et al. Hypoxic TumorDerived Exosomal miR-301a Mediates M2 Macrophage Polarization via PTEN/PI3Kgamma to Promote Pancreatic Cancer Metastasis. Cancer Res. 2018;78(16):4586-98.

160. Wang X, Luo G, Zhang K, Cao J, Huang C, Jiang T, et al. Correction: Hypoxic Tumor-Derived Exosomal miR-301a Mediates M2 Macrophage Polarization via PTEN/PI3Kgamma to Promote Pancreatic Cancer Metastasis. Cancer Res. 2020;80(4):922.

161. Cooks T, Pateras IS, Jenkins LM, Patel KM, Robles Al, Morris J, et al. Mutant p53 cancers reprogram macrophages to tumor supporting macrophages via exosomal miR-1246. Nat Commun. 2018;9(1):771.

162. DeVito NC, Plebanek MP, Theivanthiran B, Hanks BA. Role of TumorMediated Dendritic Cell Tolerization in Immune Evasion. Front Immunol. 2019;10:2876

163. Mohammadpour H, MacDonald CR, Qiao G, Chen M, Dong B, Hylander BL, et al. beta2 adrenergic receptor-mediated signaling regulates the immunosuppressive potential of myeloid-derived suppressor cells. J Clin Invest. 2019;129(12):5537-52.

164. Yang Z, Guo J, Weng L, Tang W, Jin S, Ma W. Myeloid-derived suppressor cells-new and exciting players in lung cancer. J Hematol Oncol. 2020;13(1): 10.

165. Guo X, Qiu W, Liu Q, Qian M, Wang S, Zhang Z, et al. Immunosuppressive effects of hypoxia-induced glioma exosomes through myeloid-derived suppressor cells via the miR-10a/Rora and miR-21/Pten Pathways. Oncogene. 2018;37(31):4239-59.

166. Bruns H, Bottcher M, Qorraj M, Fabri M, Jitschin S, Dindorf J, et al. CLL-cellmediated MDSC induction by exosomal miR-155 transfer is disrupted by vitamin D. Leukemia. 2017;31(4):985-8.

167. Deng Z, Rong Y, Teng Y, Zhuang X, Samykutty A, Mu J, et al. Exosomes miR-126a released from MDSC induced by DOX treatment promotes lung metastasis. Oncogene. 2017;36(5):639-51.

168. Cheng L, Sharples RA, Scicluna BJ, Hill AF. Exosomes provide a protective and enriched source of miRNA for biomarker profiling compared to intracellular and cell-free blood. J Extracell Vesicles. 2014;3.

169. Zickler AM, El Andaloussi S. Functional extracellular vesicles aplenty. Nat Biomed Eng. 2020;4(1):9-11.

170. Bhagirath D, Yang TL, Bucay N, Sekhon K, Majid S, Shahryari V, et al. microRNA-1246 Is an Exosomal Biomarker for Aggressive Prostate Cancer. Cancer Res. 2018;78(7):1833-44

171. Li XJ, Ren ZJ, Tang JH, Yu Q. Exosomal MicroRNA MiR-1246 Promotes Cell Proliferation, Invasion and Drug Resistance by Targeting CCNG2 in Breast Cancer. Cell Physiol Biochem. 2017:44(5):1741-8.

172. Sakha S, Muramatsu T, Ueda K, Inazawa J. Exosomal microRNA miR-1246 induces cell motility and invasion through the regulation of DENND2D in oral squamous cell carcinoma. Sci Rep. 2016;6:38750.
173. Zhai LY, Li MX, Pan WL, Chen Y, Li MM, Pang JX, et al. In Situ Detection of Plasma Exosomal MicroRNA-1246 for Breast Cancer Diagnostics by a Au Nanoflare Probe. ACS Appl Mater Interfaces. 2018;10(46):39478-86.

174. Meltzer S, Bjornetro T, Lyckander LG, Flatmark K, Dueland S, Samiappan R, et al. Circulating Exosomal miR-141-3p and miR-375 in Metastatic Progression of Rectal Cancer. Transl Oncol. 2019;12(8):1038-44.

175. Su YY, Sun L, Guo ZR, Li JC, Bai TT, Cai XX, et al. Upregulated expression of serum exosomal miR-375 and miR-1307 enhance the diagnostic power of CA125 for ovarian cancer. J Ovarian Res. 2019;12(1):6.

176. Foj L, Ferrer F, Serra M, Arevalo A, Gavagnach M, Gimenez N, et al. Exosomal and Non-Exosomal Urinary miRNAs in Prostate Cancer Detection and Prognosis. Prostate. 2017;77(6):573-83.

177. Zheng M, Hou L, Ma Y, Zhou L, Wang F, Cheng B, et al. Exosomal let-7d-3p and miR-30d-5p as diagnostic biomarkers for non-invasive screening of cervical cancer and its precursors. Mol Cancer. 2019;18(1):76.

178. Lee YR, Kim G, Tak WY, Jang SY, Kweon YO, Park JG, et al. Circulating exosomal noncoding RNAs as prognostic biomarkers in human hepatocellular carcinoma. Int J Cancer. 2019;144(6):1444-52.

179. Asadirad A, Hashemi SM, Baghaei K, Ghanbarian H, Mortaz E, Zali MR, et al. Phenotypical and functional evaluation of dendritic cells after exosomal delivery of miRNA-155. Life Sci. 2019;219:152-62.

180. Liang G, Zhu Y, Ali DJ, Tian T, Xu H, Si K, et al. Engineered exosomes for targeted co-delivery of miR-21 inhibitor and chemotherapeutics to reverse drug resistance in colon cancer. J Nanobiotechnology. 2020;18(1):10.

181. McAndrews KM, Kalluri R. Mechanisms associated with biogenesis of exosomes in cancer. Mol Cancer. 2019;18(1):52.

182. Jiang W, Ma P, Deng L, Liu Z, Wang X, Liu X, et al. Hepatitis A virus structural protein $\mathrm{pX}$ interacts with ALIX and promotes the secretion of virions and foreign proteins through exosome-like vesicles. J Extracell Vesicles. 2020;9(1):1716513.

183. Villarroya-Beltri C, Baixauli F, Mittelbrunn M, Fernandez-Delgado I, Torralba D, Moreno-Gonzalo O, et al. ISGylation controls exosome secretion by promoting lysosomal degradation of MVB proteins. Nat Commun. 2016;7: 13588.

184. Leidal AM, Huang HH, Marsh T, Solvik T, Zhang D, Ye J, et al. The LC3conjugation machinery specifies the loading of RNA-binding proteins into extracellular vesicles. Nat Cell Biol. 2020;22(2):187-99.

\section{Publisher's Note}

Springer Nature remains neutral with regard to jurisdictional claims in published maps and institutional affiliations.

Ready to submit your research? Choose BMC and benefit from:

- fast, convenient online submission

- thorough peer review by experienced researchers in your field

- rapid publication on acceptance

- support for research data, including large and complex data types

- gold Open Access which fosters wider collaboration and increased citations

- maximum visibility for your research: over $100 \mathrm{M}$ website views per year

At $\mathrm{BMC}$, research is always in progress.

Learn more biomedcentral.com/submissions 